\title{
Search for Gravitational Waves from a Long-lived Remnant of the Binary Neutron Star Merger GW170817
}

B. P. Abbott ${ }^{1}$, R. Abbott ${ }^{1}$, T. D. Abbott ${ }^{2}$, F. Acernese ${ }^{3,4}$, K. Ackley ${ }^{5}$ (10, C. Adams ${ }^{6}$, T. Adams $^{7}$, P. Addesso ${ }^{8}$, R. X. Adhikari ${ }^{1}$, V. B. Adya ${ }^{9,10}$, C. Affeldt ${ }^{9,10}$, B. Agarwal ${ }^{11}$, M. Agathos $^{12}$, K. Agatsuma $^{13}$, N. Aggarwal ${ }^{14}$, O. D. Aguiar ${ }^{15}$, L. Aiello ${ }^{16,17}$

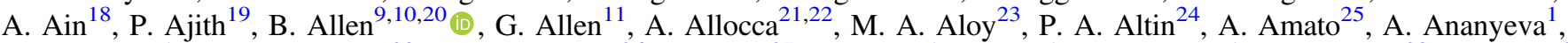
S. B. Anderson ${ }^{1}$, W. G. Anderson ${ }^{20}$, S. V. Angelova ${ }^{26}$, S. Antier ${ }^{27}$, S. Appert ${ }^{1}$, K. Arai ${ }^{1}$, M. C. Araya ${ }^{1}$, J. S. Areeda ${ }^{28}$, M. Arène ${ }^{29}$, N. Arnaud ${ }^{27,30}$, S. Ascenzi ${ }^{31,32}$, G. Ashton ${ }^{5}$, M. Ast ${ }^{33}$, S. M. Aston ${ }^{6}$, P. Astone ${ }^{34}$, D. V. Atallah ${ }^{35}$, F. Aubin ${ }^{7}$ (1) P. Aufmuth ${ }^{10}$, C. Aulbert 9 (10, K. AultONeal ${ }^{36}$, C. Austin ${ }^{2}$, A. Avila-Alvarez ${ }^{28}$, S. Babak ${ }^{29,37}$, P. Bacon ${ }^{29}$, F. Badaracco ${ }^{16,17}$, M. K. M. Bader ${ }^{13}$, S. Bae ${ }^{38}$, P. T. Baker ${ }^{39}$, F. Baldaccini ${ }^{40,41}$, G. Ballardin ${ }^{30}$, S. W. Ballmer ${ }^{42}$, S. Banagiri ${ }^{43}$, J. C. Barayoga ${ }^{1}$, S. E. Barclay ${ }^{44}$, B. C. Barish ${ }^{1}$, D. Barker ${ }^{45}$, K. Barkett ${ }^{46}$, S. Barnum ${ }^{14}$, F. Barone ${ }^{3,4}$, B. Barr ${ }^{44}$, L. Barsotti ${ }^{14}$, M. Barsuglia ${ }^{29}$, D. Barta ${ }^{47}$, J. Bartlett ${ }^{45}$,

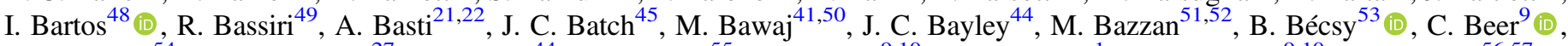
M. Bejger ${ }^{54}$ (1) I. Belahcene ${ }^{27}$, A. S. Bell ${ }^{44}$, D. Beniwal ${ }^{55}$, M. Bensch ${ }^{9,10}$, B. K. Berger ${ }^{1}$, G. Bergmann ${ }^{9,10}$, S. Bernuzzi ${ }^{56,57}$, J. J. Bero ${ }^{58}$, C. P. L. Berry ${ }^{59}$, D. Bersanetti ${ }^{60}$, A. Bertolini ${ }^{13}$, J. Betzwieser ${ }^{6}$, R. Bhandare ${ }^{61}$, I. A. Bilenko ${ }^{62}$, S. A. Bilgili ${ }^{39}$, G. Billingsley ${ }^{1}$, C. R. Billman ${ }^{48}$, J. Birch ${ }^{6}$, R. Birney ${ }^{26}$, O. Birnholtz ${ }^{58}$, S. Biscans ${ }^{1,14}$, S. Biscoveanu ${ }^{5}$, A. Bisht ${ }^{9,10}$, M. Bitossi ${ }^{22,30}$, M. A. Bizouard ${ }^{27}$, J. K. Blackburn ${ }^{1}$, J. Blackman ${ }^{46}$, C. D. Blair ${ }^{6}$, D. G. Blair ${ }^{63}$, R. M. Blair ${ }^{45}$, S. Bloemen ${ }^{64}$, O. Bock ${ }^{9}$, N. Bode ${ }^{9,10}$, M. Boer ${ }^{65}$, Y. Boetzel ${ }^{66}$, G. Bogaert ${ }^{65}$, A. Bohe ${ }^{37}$, F. Bondu ${ }^{67}$, E. Bonilla ${ }^{49}$, R. Bonnand ${ }^{7}$, P. Booker ${ }^{9,10}$, B. A. Boom ${ }^{13}$, C. D. Booth ${ }^{35}$, R. Bork ${ }^{1}$, V. Boschi ${ }^{30}$, S. Bose $^{18,68}$, K. Bossie $^{6}$, V. Bossilkov ${ }^{63}$, J. Bosveld ${ }^{63}$, Y. Bouffanais ${ }^{29}$, A. Bozzi $^{30}$, C. Bradaschia ${ }^{22}$, P. R. Brady ${ }^{20}$, A. Bramley ${ }^{6}$, M. Branchesi ${ }^{16,17}$, J. E. Brau ${ }^{69}$, T. Briant ${ }^{70}$, F. Brighenti ${ }^{71,72}$, A. Brillet ${ }^{65}$, M. Brinkmann ${ }^{9,10}$, V. Brisson ${ }^{27,168}$, P. Brockill ${ }^{20}$, A. F. Brooks ${ }^{1}$, D. D. Brown ${ }^{55}$, S. Brunett ${ }^{1}$, C. C. Buchanan ${ }^{2}$, A. Buikema ${ }^{14}$, T. Bulik ${ }^{73}$, H. J. Bulten ${ }^{13,74}$, A. Buonanno ${ }^{37,75}$, D. Buskulic ${ }^{7}$, C. Buy ${ }^{29}$, R. L. Byer ${ }^{49}$, M. Cabero 9 , L. Cadonati ${ }^{76}$, G. Cagnoli ${ }^{25,77}$, C. Cahillane ${ }^{1}$, J. Calderón Bustillo ${ }^{76}$, T. A. Callister ${ }^{1}$, E. Calloni ${ }^{4,78}$, J. B. Camp ${ }^{79}$, M. Canepa ${ }^{60,80}$, P. Canizares ${ }^{64}$, K. C. Cannon ${ }^{81}$,

H. $\mathrm{CaO}^{55}$, J. $\mathrm{CaO}^{82}$, C. D. Capano ${ }^{9}$, E. Capocasa ${ }^{29}$, F. Carbognani ${ }^{30}$, S. Caride ${ }^{83}$, M. F. Carney ${ }^{84}$, J. Casanueva Diaz ${ }^{22}$,

C. Casentini ${ }^{31,32}$, S. Caudill ${ }^{13,20}$, M. Cavaglià ${ }^{85}$, F. Cavalier ${ }^{27}$, R. Cavalieri ${ }^{30}$, G. Cella ${ }^{22}$, C. B. Cepeda ${ }^{1}$, P. Cerdá-Durán ${ }^{23}$,

G. Cerretani $^{21,22}$, E. Cesarini ${ }^{32,86}$, O. Chaibi ${ }^{65}$, S. J. Chamberlin ${ }^{87}$, M. Chan ${ }^{44}$, S. Chao ${ }^{88}$, P. Charlton ${ }^{89}$ (i), E. Chase ${ }^{90}$,

E. Chassande-Mottin ${ }^{29}$, D. Chatterjee ${ }^{20}$, B. D. Cheeseboro ${ }^{39}$, H. Y. Chen ${ }^{91}$, X. Chen ${ }^{63}$, Y. Chen ${ }^{46}$, H.-P. Cheng ${ }^{48}$, H. Y. Chia ${ }^{48}$, A. Chincarini ${ }^{60}$, A. Chiummo o ${ }^{30}$, T. Chmiel ${ }^{84}$, H. S. Cho ${ }^{92}$, M. Cho ${ }^{75}$, J. H. Chow ${ }^{24}$, N. Christensen ${ }^{65,93}$, Q. Chu ${ }^{63}$, A. J. K. Chua ${ }^{46}$,

S. Chua ${ }^{70}$, K. W. Chung ${ }^{94}$, S. Chung ${ }^{63}$, G. Ciani ${ }^{48,51,52}$, A. A. Ciobanu ${ }^{55}$, R. Ciolfi ${ }^{95,96}$ (1) , F. Cipriano ${ }^{65}$, C. E. Cirelli ${ }^{49}$,

A. Cirone ${ }^{60,80}$, F. Clara ${ }^{45}$, J. A. Clark ${ }^{76}$, P. Clearwater ${ }^{97}$, F. Cleva ${ }^{65}$, C. Cocchieri ${ }^{85}$, E. Coccia ${ }^{16,17}$, P.-F. Cohadon ${ }^{70}$, D. Cohen ${ }^{27}$, A. Colla ${ }^{34,98}$, C. G. Collette ${ }^{99}$, C. Collins ${ }^{59}$, L. R. Cominsky ${ }^{100}$, M. Constancio, Jr. ${ }^{15}$, L. Conti ${ }^{52}$, S. J. Cooper ${ }^{59}$, P. Corban ${ }^{6}$, T. R. Corbitt ${ }^{2}$, I. Cordero-Carrión ${ }^{101}$, K. R. Corley ${ }^{102}$, N. Cornish ${ }^{103}$ (1) A. Corsi ${ }^{83}$ (i), S. Cortese ${ }^{30}$, C. A. Costa ${ }^{15}$, R. Cotesta ${ }^{37}$, M. W. Coughlin ${ }^{1}$ (D, S. B. Coughlin ${ }^{35,90}$, J.-P. Coulon ${ }^{65}$, S. T. Countryman ${ }^{102}$, P. Couvares ${ }^{1}$, P. B. Covas ${ }^{104}$, E. E. Cowan ${ }^{76}$, D. M. Coward ${ }^{63}$, M. J. Cowart ${ }^{6}$, D. C. Coyne ${ }^{1}$, R. Coyne ${ }^{105}$, J. D. E. Creighton ${ }^{20}$, T. D. Creighton ${ }^{106}$, J. Cripe ${ }^{2}$, S. G. Crowder ${ }^{107}$, T. J. Cullen ${ }^{2}$, A. Cumming ${ }^{44}$, L. Cunningham ${ }^{44}$, E. Cuoco ${ }^{30}$, T. Dal Canton ${ }^{79}$ (1) , G. Dálya ${ }^{53}$, S. L. Danilishin ${ }^{9,10}$, S. D'Antonio ${ }^{32}$, K. Danzmann ${ }^{9,10}$, A. Dasgupta ${ }^{108}$, C. F. Da Silva Costa ${ }^{48}$, V. Dattilo ${ }^{30}$, I. Dave ${ }^{61}$, M. Davier ${ }^{27}$, D. Davis ${ }^{42}$, E. J. Daw ${ }^{109}$, B. Day $^{76}$, D. DeBra ${ }^{49}$, M. Deenadayalan ${ }^{18}$, J. Degallaix ${ }^{25}$, M. De Laurentis ${ }^{4,78}$, S. Deléglise ${ }^{70}$, W. Del Pozzo ${ }^{21,22}$, N. Demos ${ }^{14}$, T. Denker ${ }^{9}, 10$, T. Dent ${ }^{9}$ (1), R. De Pietri ${ }^{56,57}$, J. Derby ${ }^{28}$, V. Dergachev ${ }^{9}$, R. De Rosa ${ }^{4,78}$, C. De Rossi ${ }^{25,30}$, R. DeSalvo ${ }^{110}$, O. de Varona ${ }^{9,10}$, S. Dhurandhar ${ }^{18}$, M. C. Díaz ${ }^{106}$, L. Di Fiore ${ }^{4}$, M. Di Giovanni ${ }^{96,111}$, T. Di Girolamo ${ }^{4,78}$, A. Di Lieto ${ }^{21,22}$, B. Ding ${ }^{99}$, S. Di Pace ${ }^{34,98}$, I. Di Palma ${ }^{34,98}$, F. Di Renzo ${ }^{21,22}$, A. Dmitriev ${ }^{59}$, Z. Doctor ${ }^{91}$ (1) , V. Dolique ${ }^{25}$, F. Donovan ${ }^{14}$, K. L. Dooley ${ }^{35,85}$, S. Doravari ${ }^{9,10}$, I. Dorrington ${ }^{35}$, M. Dovale Álvarez ${ }^{59}$, T. P. Downes ${ }^{20}$, M. Drago, 9 ,16,17 C. Dreissigacker ${ }^{9,10}$, J. C. Driggers ${ }^{45}$, Z. Du ${ }^{82}$, P. Dupej $^{44}$, S. E. Dwyer ${ }^{45}$, P. J. Easter ${ }^{5}$, T. B. Edo ${ }^{109}$, M. C. Edwards $^{93}$, A. Effler ${ }^{6}$, H.-B. Eggenstein ${ }^{9,10}$ (i), P. Ehrens ${ }^{1}$, J. Eichholz ${ }^{1}$, S. S. Eikenberry ${ }^{48}$, M. Eisenmann ${ }^{7}$, R. A. Eisenstein ${ }^{14}$, R. C. Essick $^{91}$, H. Estelles ${ }^{104}$, D. Estevez ${ }^{7}$, Z. B. Etienne ${ }^{39}$, T. Etzel ${ }^{1}$, M. Evans ${ }^{14}$, T. M. Evans ${ }^{6}$, V. Fafone ${ }^{16,31,32}$, H. Fair ${ }^{42}$, S. Fairhurst ${ }^{35}$ (1), X. Fan ${ }^{82}$, S. Farinon ${ }^{60}$, B. Farr ${ }^{69}$ (1), W. M. Farr ${ }^{59}$ (1), E. J. Fauchon-Jones ${ }^{35}$, M. Favata ${ }^{112}$, M. Fays ${ }^{35}$, C. Fee ${ }^{84}$, H. Fehrmann ${ }^{9}$, J. Feicht ${ }^{1}$, M. M. Fejer ${ }^{49}$, F. Feng ${ }^{29}$,

A. Fernandez-Galiana ${ }^{14}$, I. Ferrante ${ }^{21,22}$, E. C. Ferreira ${ }^{15}$, F. Ferrini ${ }^{30}$, F. Fidecaro ${ }^{21,22}$, I. Fiori ${ }^{30}$, D. Fiorucci ${ }^{29}$, M. Fishbach ${ }^{91}$ (D), R. P. Fisher ${ }^{42}$, J. M. Fishner ${ }^{14}$, M. Fitz-Axen ${ }^{43}$, R. Flaminio ${ }^{7,113}$, M. Fletcher ${ }^{44}$, H. Fong ${ }^{114}$, J. A. Font ${ }^{23,115}$, P. W. F. Forsyth ${ }^{24}$, S. S. Forsyth ${ }^{76}$, J.-D. Fournier ${ }^{65}$, S. Frasca ${ }^{34,98}$, F. Frasconi ${ }^{22}$, Z. Frei $^{53}$, A. Freise ${ }^{59}$, R. Frey ${ }^{69}$, V. Frey ${ }^{27}$, P. Fritschel ${ }^{14}$, V. V. Frolov ${ }^{6}$, P. Fulda ${ }^{48}$, M. Fyffe ${ }^{6}$, H. A. Gabbard ${ }^{44}$, B. U. Gadre ${ }^{18}$, S. M. Gaebel ${ }^{59}$, J. R. Gair ${ }^{116}$, L. Gammaitoni ${ }^{40}$, M. R. Ganija ${ }^{55}$, S. G. Gaonkar ${ }^{18}$, A. Garcia ${ }^{28}$, C. García-Quirós ${ }^{104}$, F. Garufi ${ }^{4,78}$, B. Gateley ${ }^{45}$, S. Gaudio ${ }^{36}$, G. Gaur ${ }^{117}$, V. Gayathri ${ }^{118}$, G. Gemme ${ }^{60}$, E. Genin ${ }^{30}$, A. Gennai ${ }^{22}$, D. George ${ }^{11}$, J. George ${ }^{61}$, L. Gergely ${ }^{119}$, V. Germain ${ }^{7}$, S. Ghonge ${ }^{76}$, Abhirup Ghosh ${ }^{19}$, Archisman Ghosh ${ }^{13}$, S. Ghosh ${ }^{20}$, B. Giacomazzo ${ }^{96,111}$ (i), J. A. Giaime ${ }^{2,6}$, K. D. Giardina ${ }^{6}$, A. Giazotto 22,169, K. Gill ${ }^{36}$, G. Giordano ${ }^{3,4}$, L. Glover ${ }^{110}$, E. Goetz ${ }^{45}$, R. Goetz ${ }^{48}$, B. Goncharov ${ }^{5}$, G. González ${ }^{2} \oplus$, J. M. Gonzalez Castro ${ }^{21,22}$, A. Gopakumar ${ }^{120}$, M. L. Gorodetsky ${ }^{62}$, S. E. Gossan ${ }^{1}$, M. Gosselin ${ }^{30}$, R. Gouaty ${ }^{7}$, A. Grado ${ }^{4,12}$ (i), C. Graef ${ }^{44}$, M. Granata ${ }^{25}$, A. Grant ${ }^{44}$, S. Gras ${ }^{14}$, C. Gray ${ }^{45}$, G. Greco ${ }^{71,72}$, A. C. Green ${ }^{59}$, R. Green ${ }^{35}$, E. M. Gretarsson ${ }^{36}$, P. Groot ${ }^{64}$, H. Grote $^{35}$, S. Grunewald ${ }^{37}$, P. Gruning ${ }^{27}$, G. M. Guidi ${ }^{71,72}$, H. K. Gulati ${ }^{108}$, X. Guo ${ }^{82}$, A. Gupta ${ }^{87}$, M. K. Gupta ${ }^{108}$, K. E. Gushwa ${ }^{1}$, 
E. K. Gustafson ${ }^{1}$, R. Gustafson ${ }^{122}$, O. Halim ${ }^{16,17}$, B. R. Hall ${ }^{68}$, E. D. Hall ${ }^{14}$, E. Z. Hamilton ${ }^{35}$, H. F. Hamilton ${ }^{123}$, G. Hammond ${ }^{44}$, M. Haney ${ }^{66}$, M. M. Hanke ${ }^{9,10}$, J. Hanks ${ }^{45}$, C. Hanna ${ }^{87}$, M. D. Hannam ${ }^{35}$, O. A. Hannuksela ${ }^{94}$, J. Hanson ${ }^{6}$, T. Hardwick ${ }^{2}$, J. Harms ${ }^{16,17}$, G. M. Harry ${ }^{124}$, I. W. Harry ${ }^{37}$, M. J. Hart ${ }^{44}$, C.-J. Haster ${ }^{14}$ (i), K. Haughian ${ }^{44}$, J. Healy ${ }^{58}$, A. Heidmann ${ }^{70}$, M. C. Heintze ${ }^{6}$, H. Heitmann ${ }^{65}$, P. Hello ${ }^{27}$, G. Hemming ${ }^{30}$, M. Hendry ${ }^{44}$, I. S. Heng ${ }^{44}$ (10, J. Hennig ${ }^{44}$, A. W. Heptonstall ${ }^{1}$, F. J. Hernandez ${ }^{5}$, M. Heurs ${ }^{9,10}$, S. Hild ${ }^{44}$, T. Hinderer ${ }^{64}$, D. Hoak ${ }^{30}$, S. Hochheim ${ }^{9,10}$, D. Hofman ${ }^{25}$, N. A. Holland ${ }^{24}$, K. Holt ${ }^{6}$, D. E. Holz ${ }^{91}$ (1) P. Hopkins ${ }^{35}$, C. Horst ${ }^{20}$, J. Hough ${ }^{44}$, E. A. Houston ${ }^{44}$, E. J. Howell ${ }^{63}$, A. Hreibi ${ }^{65}$, E. A. Huerta ${ }^{11}$, D. Huet ${ }^{27}$,

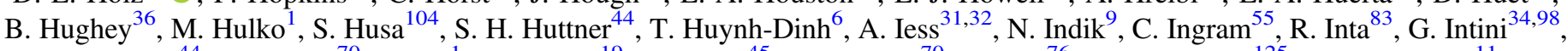

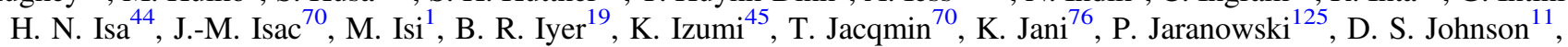

W. W. Johnson ${ }^{2}$, D. I. Jones ${ }^{126}$, R. Jones ${ }^{44}$, R. J. G. Jonker ${ }^{13}$, L. Ju ${ }^{63}$, J. Junker ${ }^{9}, 10$, C. V. Kalaghatgi ${ }^{35}$, V. Kalogera ${ }^{90}$ (1) , B. Kamai ${ }^{1}$, S. Kandhasamy ${ }^{6}$, G. Kang ${ }^{38}$, J. B. Kanner ${ }^{1}$, S. J. Kapadia ${ }^{20}$, S. Karki ${ }^{69}$, K. S. Karvinen ${ }^{910}$, M. Kasprzack ${ }^{2}$, W. Kastaun ${ }^{9}$, M. Katolik ${ }^{11}$, S. Katsanevas ${ }^{30}$, E. Katsavounidis ${ }^{14}$, W. Katzman ${ }^{6}$, S. Kaufer ${ }^{9,10}$, K. Kawabe ${ }^{45}$, N. V. Keerthana ${ }^{18}$, F. Kéfélian ${ }^{65}$, D. Keitel ${ }^{44}$ (1) , A. J. Kemball ${ }^{11}$, R. Kennedy ${ }^{109}$, J. S. Key ${ }^{127}$, F. Y. Khalili ${ }^{62}$, B. Khamesra ${ }^{76}$, H. Khan ${ }^{28}$, I. Khan ${ }^{16,32}$, S. Khan ${ }^{9}$, Z. Khan ${ }^{108}$, E. A. Khazanov ${ }^{128}$, N. Kijbunchoo ${ }^{24}$, Chunglee $\mathrm{Kim}^{129}$, J. C. Kim ${ }^{130}$, K. Kim ${ }^{94}$, W. Kim ${ }^{55}$, W. S. Kim ${ }^{131}$, Y.-M. Kim ${ }^{132}$, E. J. King ${ }^{55}$, P. J. King ${ }^{45}$, M. Kinley-Hanlon ${ }^{124}$, R. Kirchhoff ${ }^{9,10}$, J. S. Kissel ${ }^{45}$, L. Kleybolte ${ }^{33}$, S. Klimenko ${ }^{48}$, T. D. Knowles ${ }^{39}$, P. Koch ${ }^{9,10}$ (10, S. M. Koehlenbeck ${ }^{9,10}$, S. Koley ${ }^{13}$, V. Kondrashov ${ }^{1}$, A. Kontos ${ }^{14}$, M. Korobko ${ }^{33}$, W. Z. Korth ${ }^{1}$, I. Kowalska $^{73}$ (10), D. B. Kozak ${ }^{1}$, C. Krämer ${ }^{9}$, V. Kringel ${ }^{9,10}$, B. Krishnan ${ }^{9}$ (i), A. Królak ${ }^{133,134}$, G. Kuehn ${ }^{9,10}$, P. Kumar ${ }^{135}$, R. Kumar $^{108}$, S. Kumar ${ }^{19}$, L. Kuo ${ }^{88}$, A. Kutynia ${ }^{133}$, S. Kwang ${ }^{20}$, B. D. Lackey ${ }^{37}$, K. H. Lai ${ }^{94}$, M. Landry ${ }^{45}$, R. N. Lang ${ }^{136}$, J. Lange ${ }^{58}$ (1) B. Lantz $^{49}$, R. K. Lanza ${ }^{14}$, A. Lartaux-Vollard ${ }^{27}$, P. D. Lasky ${ }^{5}$ (1), M. Laxen ${ }^{6}$, A. Lazzarini ${ }^{1}$, C. Lazzaro ${ }^{52}$, P. Leaci ${ }^{34,98}$, S. Leavey ${ }^{9,10}$, C. H. Lee ${ }^{92}$, H. K. Lee ${ }^{137}$, H. M. Lee ${ }^{129}$, H. W. Lee ${ }^{130}$, K. Lee ${ }^{44}$, J. Lehmann ${ }^{9,10}$, A. Lenon ${ }^{39}$, M. Leonardi ${ }^{9}, 10,113$, N. Leroy ${ }^{27}$, N. Letendre ${ }^{7}$, Y. Levin ${ }^{5}$, J. Li ${ }^{82}$, T. G. F. Li ${ }^{94}$, X. Li ${ }^{46}$, S. D. Linker ${ }^{110}$, T. B. Littenberg ${ }^{138}$, J. Liu $^{63}$, X. Liu ${ }^{20}$, R. K. L. Lo ${ }^{94}$, N. A. Lockerbie ${ }^{26}$, L. T. London ${ }^{35}$, A. Longo ${ }^{139,140}$, M. Lorenzini ${ }^{16,17}$, V. Loriette ${ }^{141}$, M. Lormand ${ }^{6}$, G. Losurdo ${ }^{22}$, J. D. Lough, ${ }^{9,10}$, C. O. Lousto ${ }^{58}$ (1) , G. Lovelace ${ }^{28}$, H. Lück ${ }^{9,10}$, D. Lumaca ${ }^{31,32}$, A. P. Lundgren ${ }^{9}$, R. Lynch ${ }^{14}$, Y. Ma ${ }^{46}$, R. Macas ${ }^{35}$, S. Macfoy ${ }^{26}$, B. Machenschalk ${ }^{9}$, M. MacInnis ${ }^{14}$, D. M. Macleod ${ }^{35}$, I. Magaña Hernandez ${ }^{20}$,

F. Magaña-Sandoval ${ }^{42}$, L. Magaña Zertuche ${ }^{85}$, R. M. Magee ${ }^{87}$, E. Majorana ${ }^{34}$, I. Maksimovic ${ }^{141}$, N. Man ${ }^{65}$, V. Mandic ${ }^{43}$, V. Mangano ${ }^{44}$, G. L. Mansell ${ }^{24}$, M. Manske ${ }^{20,24}$, M. Mantovani ${ }^{30}$, F. Marchesoni $^{41,50}$, F. Marion ${ }^{7}$, S. Márka ${ }^{102}$, Z. Márka $^{102}$, C. Markakis ${ }^{11}$, A. S. Markosyan ${ }^{49}$, A. Markowitz ${ }^{1}$, E. Maros ${ }^{1}$, A. Marquina ${ }^{101}$, F. Martelli ${ }^{71,72}$, L. Martellini ${ }^{65}$, I. W. Martin ${ }^{44}$,

R. M. Martin ${ }^{112}$, D. V. Martynov ${ }^{14}$, K. Mason ${ }^{14}$, E. Massera ${ }^{109}$, A. Masserot ${ }^{7}$, T. J. Massinger ${ }^{1}$, M. Masso-Reid ${ }^{44}$,

S. Mastrogiovanni ${ }^{34,98}$, A. Matas ${ }^{43}$, F. Matichard ${ }^{1,14}$, L. Matone ${ }^{102}$, N. Mavalvala ${ }^{14}$, N. Mazumder ${ }^{68}$, J. J. McCann ${ }^{63}$, R. McCarthy ${ }^{45}$, D. E. McClelland ${ }^{24}$, S. McCormick ${ }^{6}$, L. McCuller $^{14}$, S. C. McGuire ${ }^{142}$, J. McIver ${ }^{1}$, D. J. McManus ${ }^{24}$, T. McRae $^{24}$, S. T. McWilliams ${ }^{39}$, D. Meacher ${ }^{87}$, G. D. Meadors ${ }^{5}$, M. Mehmet ${ }^{9,10}$, J. Meidam ${ }^{13}$, E. Mejuto-Villa ${ }^{8}$, A. Melatos ${ }^{97}$, G. Mendell ${ }^{45}$, D. Mendoza-Gandara ${ }^{9,10}$, R. A. Mercer ${ }^{20}$, L. Mereni ${ }^{25}$, E. L. Merilh ${ }^{45}$, M. Merzougui ${ }^{65}$, S. Meshkov ${ }^{1}$, C. Messenger ${ }^{44}$,

C. Messick ${ }^{87}$ (10), R. Metzdorff ${ }^{70}$, P. M. Meyers ${ }^{43}$, H. Miao ${ }^{59}$, C. Michel ${ }^{25}$, H. Middleton ${ }^{97}$, E. E. Mikhailov ${ }^{143}$, L. Milano ${ }^{4,78}$, A. L. Miller ${ }^{48}$, A. Miller ${ }^{34,98}$, B. B. Miller ${ }^{90}$ J. Miller $^{14}$, M. Millhouse ${ }^{103}$, J. Mills ${ }^{35}$, M. C. Milovich-Goff ${ }^{110}$, O. Minazzoli ${ }^{65,144}$, Y. Minenkov ${ }^{32}$, J. Ming ${ }^{9,10}$, C. Mishra ${ }^{145}$, S. Mitra ${ }^{18}$, V. P. Mitrofanov ${ }^{62}$, G. Mitselmakher ${ }^{48}$, R. Mittleman ${ }^{14}$, D. Moffa ${ }^{84}$, K. Mogushi ${ }^{85}$ (1), M. Mohan ${ }^{30}$, S. R. P. Mohapatra ${ }^{14}$, M. Montani ${ }^{71,72}$, C. J. Moore ${ }^{12}$, D. Moraru ${ }^{45}$, G. Moreno ${ }^{45}$, S. Morisaki ${ }^{81}$, B. Mours ${ }^{7}$, C. M. Mow-Lowry ${ }^{59}$, G. Mueller ${ }^{48}$, A. W. Muir ${ }^{35}$, Arunava Mukherjee ${ }^{9,10}$, D. Mukherjee ${ }^{20}$, S. Mukherjee ${ }^{106}$, N. Mukund ${ }^{18}$, A. Mullavey ${ }^{6}$, J. Munch ${ }^{55}$, E. A. Muñiz ${ }^{42}$, M. Muratore ${ }^{36}$, P. G. Murray ${ }^{44}$, A. Nagar ${ }^{86,146,147}$, K. Napier ${ }^{76}$, I. Nardecchia ${ }^{31,32}$, L. Naticchioni ${ }^{34,98}$, R. K. Nayak ${ }^{148}$, J. Neilson ${ }^{110}$, G. Nelemans ${ }^{13,64}$, T. J. N. Nelson ${ }^{6}$, M. Nery ${ }^{9,10}$, A. Neunzert ${ }^{122}$, L. Nevin ${ }^{1}$, J. M. Newport ${ }^{124}$, K. Y. $\mathrm{Ng}^{14}, \mathrm{~S}^{14} \mathrm{Ng}^{55}, \mathrm{P}$. Nguyen $^{69}$, T. T. Nguyen ${ }^{24}$, D. Nichols ${ }^{64}$, A. B. Nielsen ${ }^{9}$, S. Nissanke ${ }^{13,64}$, A. Nitz ${ }^{9}$, F. Nocera ${ }^{30}$, D. Nolting ${ }^{6}$, C. North ${ }^{35}$, L. K. Nuttall ${ }^{35}$, M. Obergaulinger ${ }^{23}$ (D) J. Oberling ${ }^{45}$,

B. D. O’Brien ${ }^{48}$, G. D. O’Dea ${ }^{110}$, G. H. Ogin ${ }^{149}$, J. J. Oh ${ }^{131}$, S. H. Oh ${ }^{131}$, F. Ohme ${ }^{9}$, H. Ohta ${ }^{81}$, M. A. Okada ${ }^{15}$, M. Oliver ${ }^{104}$, P. Oppermann ${ }^{9,10}$, Richard J. Oram ${ }^{6}$, B. O'Reilly ${ }^{6}$, R. Ormiston ${ }^{43}$, L. F. Ortega ${ }^{48}$, R. O'Shaughnessy ${ }^{58}$ (1), S. Ossokine ${ }^{37}$, D. J. Ottaway ${ }^{55}$, H. Overmier ${ }^{6}$, B. J. Owen ${ }^{83}$ (1) A. E. Pace ${ }^{87}$, G. Pagano ${ }^{21,22}$, J. Page ${ }^{138}$, M. A. Page ${ }^{63}$, A. Pai ${ }^{118}$, S. A. Pai ${ }^{61}$, J. R. Palamos ${ }^{69}$, O. Palashov ${ }^{128}$, C. Palomba ${ }^{34}$, A. Pal-Singh ${ }^{33}$, Howard Pan ${ }^{88}$, Huang-Wei Pan ${ }^{88}$, B. Pang ${ }^{46}$, P. T. H. Pang ${ }^{94}$,

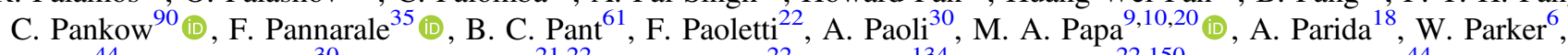

D. Pascucci ${ }^{44}$, A. Pasqualetti ${ }^{30}$, R. Passaquieti ${ }^{21,22}$, D. Passuello ${ }^{22}$, M. Patil ${ }^{134}$, B. Patricelli ${ }^{22,150}$, B. L. Pearlstone ${ }^{44}$, C. Pedersen $^{35}$, M. Pedraza ${ }^{1}$, R. Pedurand ${ }^{25,151}$, L. Pekowsky ${ }^{42}$, A. Pele ${ }^{6}$, S. Penn $^{152}$, C. J. Perez ${ }^{45}$, A. Perreca ${ }^{96,111}$, L. M. Perri ${ }^{90}$, H. P. Pfeiffer ${ }^{37,114}$, M. Phelps ${ }^{44}$, K. S. Phukon ${ }^{18}$, O. J. Piccinni ${ }^{34,98}$, M. Pichot $^{65}$, F. Piergiovanni ${ }^{71,72}$, V. Pierro ${ }^{8}$, G. Pillant ${ }^{30}$, L. Pinard ${ }^{25}$, I. M. Pinto ${ }^{8}$, M. Pirello ${ }^{45}$, M. Pitkin ${ }^{44}{ }^{1}$, R. Poggiani $^{21,22}$, P. Popolizio ${ }^{30}$, E. K. Porter ${ }^{29}$, L. Possenti ${ }^{72,153}{ }^{15}$, A. Post ${ }^{9}$, J. Powell ${ }^{154}$, J. Prasad $^{18}$, J. W. W. Pratt ${ }^{36}$, G. Pratten ${ }^{104}$, V. Predoi ${ }^{35}$, T. Prestegard ${ }^{20}$, M. Principe ${ }^{8}$, S. Privitera ${ }^{37}$, G. A. Prodi ${ }^{96,111}$, L. G. Prokhorov ${ }^{62}$, O. Puncken, ${ }^{9,10}$, M. Punturo ${ }^{41}$, P. Puppo ${ }^{34}$, M. Pürrer ${ }^{37}$, H. Qi $^{20}$, V. Quetschke $^{106}$, E. A. Quintero ${ }^{1}$,

R. Quitzow-James ${ }^{69}$, F. J. Raab ${ }^{45}$, D. S. Rabeling ${ }^{24}$, H. Radkins ${ }^{45}$, P. Raffai ${ }^{53}$ (i), S. Raja ${ }^{61}$, C. Rajan ${ }^{61}$, B. Rajbhandari ${ }^{83}$, M. Rakhmanov ${ }^{106}$, K. E. Ramirez ${ }^{106}$, A. Ramos-Buades ${ }^{104}$, Javed Rana ${ }^{18}$ (1), P. Rapagnani ${ }^{34,98}$, V. Raymond ${ }^{35}$, M. Razzano ${ }^{21,22}$ (1),

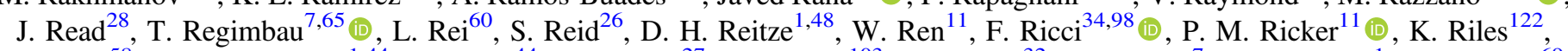
M. Rizzo ${ }^{58}$, N. A. Robertson ${ }^{1,44}$, R. Robie ${ }^{44}$, F. Robinet ${ }^{27}$, T. Robson ${ }^{103}$, A. Rocchi ${ }^{32}$, L. Rolland ${ }^{7}$, J. G. Rollins ${ }^{1}$, V. J. Roma ${ }^{69}$, R. Romano $^{3,4}$, C. L. Romel ${ }^{45}$, J. H. Romie ${ }^{6}$, D. Rosińska ${ }^{54,155}$, M. P. Ross ${ }^{156}$, S. Rowan ${ }^{44}$, A. Rüdiger ${ }^{1,10}$, P. Ruggi ${ }^{30}$, G. Rutins ${ }^{157}$, K. Ryan ${ }^{45}$, S. Sachdev ${ }^{1}$, T. Sadecki ${ }^{45}$, M. Sakellariadou ${ }^{158}$, L. Salconi ${ }^{30}$, M. Saleem ${ }^{118}$, F. Salemi ${ }^{9}$, A. Samajdar ${ }^{13,148}$, L. Sammut ${ }^{5}$, 
L. M. Sampson ${ }^{90}$, E. J. Sanchez ${ }^{1}$, L. E. Sanchez ${ }^{1}$, N. Sanchis-Gual ${ }^{23}$, V. Sandberg ${ }^{45}$, J. R. Sanders ${ }^{42}$, N. Sarin ${ }^{5}$ (1), B. Sassolas ${ }^{25}$,

B. S. Sathyaprakash ${ }^{35,87}$, P. R. Saulson ${ }^{42}$, O. Sauter ${ }^{122}$, R. L. Savage ${ }^{45}$, A. Sawadsky ${ }^{33}$, P. Schale ${ }^{69}$, M. Scheel ${ }^{46}$, J. Scheuer ${ }^{90}$, P. Schmidt ${ }^{64}$, R. Schnabel ${ }^{33}$, R. M. S. Schofield ${ }^{69}$, A. Schönbeck ${ }^{33}$, E. Schreiber ${ }^{9,10}$, D. Schuette ${ }^{9,10}$, B. W. Schulte, ${ }^{9,10}$, B. F. Schutz ${ }^{9,35}$, S. G. Schwalbe ${ }^{36}$, J. Scott ${ }^{44}$, S. M. Scott ${ }^{24}$, E. Seidel ${ }^{11}$, D. Sellers ${ }^{6}$, A. S. Sengupta ${ }^{159}$, D. Sentenac ${ }^{30}$, V. Sequino ${ }^{16,31,32}$, A. Sergeev ${ }^{128}$, Y. Setyawati ${ }^{\text {, D. A. Shaddock }}{ }^{24}$, T. J. Shaffer ${ }^{45}$, A. A. Shah ${ }^{138}$, M. S. Shahriar ${ }^{90}$, M. B. Shaner ${ }^{110}$, L. Shao ${ }^{37}$, B. Shapiro ${ }^{49}$, P. Shawhan ${ }^{75}$, H. Shen $^{11}$, D. H. Shoemaker ${ }^{14}$, D. M. Shoemaker ${ }^{76}$, K. Siellez $^{76}$, X. Siemens ${ }^{20}$, M. Sieniawska ${ }^{54}$, D. Sigg ${ }^{45}$, A. D. Silva ${ }^{15}$, L. P. Singer ${ }^{79}$, A. Singh ${ }^{9,10}$, A. Singhal ${ }^{16,34}$, A. M. Sintes ${ }^{104}$, B. J. J. Slagmolen ${ }^{24}$, T. J. Slaven-Blair ${ }^{63}$, B. Smith 6 , J. R. Smith ${ }^{28}$, R. J. E. Smith ${ }^{5}$, S. Somala ${ }^{160}$, E. J. Son ${ }^{131}$, B. Sorazu ${ }^{44}$, F. Sorrentino ${ }^{60}$, T. Souradeep ${ }^{18}$, A. P. Spencer ${ }^{44}$, A. K. Srivastava ${ }^{108}$, K. Staats ${ }^{36}$, M. Steinke ${ }^{9,10}$, J. Steinlechner ${ }^{33,44}$, S. Steinlechner ${ }^{33}$, D. Steinmeyer ${ }^{9,10}$, B. Steltner ${ }^{9,10}$, S. P. Stevenson ${ }^{154}$, D. Stocks ${ }^{49}$, R. Stone ${ }^{106}$, D. J. Stops ${ }^{59}$, K. A. Strain ${ }^{44}$, G. Stratta ${ }^{71,72}$, S. E. Strigin ${ }^{62}$, A. Strunk ${ }^{45}$, R. Sturani ${ }^{161}$, A. L. Stuver ${ }^{162}$, T. Z. Summerscales ${ }^{163}$, L. Sun ${ }^{97}$, S. Sunil ${ }^{108}$, J. Suresh ${ }^{18}$, P. J. Sutton ${ }^{35}$, B. L. Swinkels ${ }^{13}$, M. J. Szczepańczyk ${ }^{36}$, M. Tacca ${ }^{13}$, S. C. Tait ${ }^{44}$, C. Talbot ${ }^{5}$ (1) D. Talukder ${ }^{69}$, D. B. Tanner ${ }^{48}$, M. Tápai ${ }^{119}$, A. Taracchini ${ }^{37}$, J. D. Tasson ${ }^{93}$, J. A. Taylor ${ }^{138}$, R. Taylor ${ }^{1}$, S. V. Tewari ${ }^{152}$, T. Theeg ${ }^{9,10}$, F. Thies $^{9,10}$,

E. G. Thomas ${ }^{59}$, M. Thomas ${ }^{6}$, P. Thomas ${ }^{45}$, K. A. Thorne ${ }^{6}$, E. Thrane ${ }^{5}$, S. Tiwari ${ }^{16,96}$, V. Tiwari ${ }^{35}$, K. V. Tokmakov ${ }^{26}$, K. Toland $^{44}$, M. Tonelli ${ }^{21,22}$, Z. Tornasi $^{44}$, A. Torres-Forné ${ }^{23}$, C. I. Torrie ${ }^{1}$, D. Töyrä ${ }^{59}$, F. Travasso ${ }^{30,41}$, G. Traylor ${ }^{6}$, J. Trinastic ${ }^{48}$,

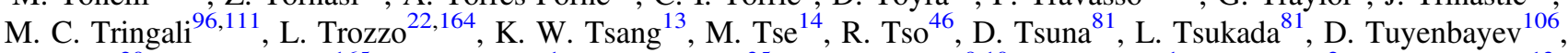
K. Ueno ${ }^{20}$, , D. Ugolini ${ }^{165}$, A. L. Urban ${ }^{1}$, S. A. Usman ${ }^{35}$, H. Vahlbruch ${ }^{9,10}$, G. Vajente ${ }^{1}$, G. Valdes ${ }^{2}$, N. van Bakel ${ }^{13}$, M. van Beuzekom ${ }^{13}$, J. F. J. van den Brand ${ }^{13,74}$, C. Van Den Broeck ${ }^{13,166}$, D. C. Vander-Hyde ${ }^{42}$, L. van der Schaaf ${ }^{13}$, J. V. van Heijningen ${ }^{13}$, A. A. van Veggel ${ }^{44}$, M. Vardaro ${ }^{51,52}$, V. Varma ${ }^{46}$, S. Vass ${ }^{1}$, M. Vasúth ${ }^{47}$, A. Vecchio ${ }^{59}$, G. Vedovato ${ }^{52}$, J. Veitch ${ }^{44}$, P. J. Veitch ${ }^{55}$, K. Venkateswara ${ }^{156}$, G. Venugopalan ${ }^{1}$, D. Verkindt', F. Vetrano ${ }^{71,72}$, A. Vicere ${ }^{71,72}$, A. D. Viets ${ }^{20}$, S. Vinciguerra ${ }^{59}$, D. J. Vine ${ }^{157}$, J.-Y. Vinet ${ }^{65}$, S. Vitale ${ }^{14}$, T. Vo $^{42}$, H. Vocca $^{40,41}$, C. Vorvick $^{45}$, S. P. Vyatchanin ${ }^{62}$, A. R. Wade ${ }^{1}$, L. E. Wade ${ }^{84}$, M. Wade ${ }^{84}$, R. Walet ${ }^{13}$, M. Walker ${ }^{28}$, L. Wallace ${ }^{1}$, S. Walsh ${ }^{9,20}$, G. Wang ${ }^{16,22}$, H. Wang ${ }^{59}$, J. Z. Wang ${ }^{122}$, W. H. Wang ${ }^{106}$, Y. F. Wang ${ }^{94}$, R. L. Ward ${ }^{24}$, J. Warner ${ }^{45}$, M. Was ${ }^{7}$, J. Watchi ${ }^{99}$, B. Weaver ${ }^{45}$, L.-W. Wei ${ }^{9}, 1^{9}$, M. Weinert ${ }^{9,10}$, A. J. Weinstein ${ }^{1}$, R. Weiss ${ }^{14}$, F. Wellmann ${ }^{9,10}$, L. Wen ${ }^{63}$, E. K. Wessel ${ }^{11}$, P. Weßels ${ }^{9,10}$, J. Westerweck ${ }^{9}$, K. Wette ${ }^{24}$, J. T. Whelan ${ }^{58}$, B. F. Whiting ${ }^{48}$, C. Whittle ${ }^{14}$, D. Wilken ${ }^{9,10}$, D. Williams ${ }^{44}$, R. D. Williams ${ }^{1}$, A. R. Williamson ${ }^{58,64}$,

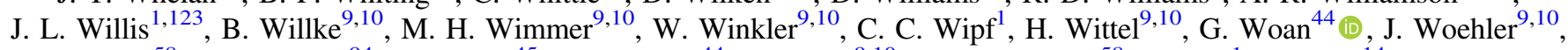
J. K. Wofford ${ }^{58}$, W. K. Wong ${ }^{94}$, J. Worden ${ }^{45}$, J. L. Wright ${ }^{44}$, D. S. Wu ${ }^{9,10}$, D. M. Wysocki ${ }^{58}$, S. Xiao ${ }^{1}$, W. Yam $^{14}$, H. Yamamoto $^{1}$, C. C. Yancey ${ }^{75}$, L. Yang ${ }^{167}$, M. J. Yap ${ }^{24}$, M. Yazback ${ }^{48}$, Hang Yu ${ }^{14}$ (1) Haocun Yu ${ }^{14}$, M. Yvert ${ }^{7}$, A. Zadrożny ${ }^{133}$, M. Zanolin $^{36}$, T. Zelenova ${ }^{30}$, J.-P. Zendri ${ }^{52}$, M. Zevin ${ }^{90}$ (1) J. Zhang ${ }^{63}$, L. Zhang ${ }^{1}$, M. Zhang ${ }^{143}$, T. Zhang ${ }^{44}$, Y.-H. Zhang ${ }^{9,10}$, C. Zhao ${ }^{63}$, M. Zhou ${ }^{90}$, Z. Zhou ${ }^{90}$, S. J. Zhu ${ }^{9,10}$, X. J. Zhu ${ }^{5}$, A. B. Zimmerman ${ }^{14}$, M. E. Zucker ${ }^{1,14}$, and J. Zweizig ${ }^{1}$

The LIGO Scientific Collaboration and the Virgo Collaboration

${ }^{1}$ LIGO, California Institute of Technology, Pasadena, CA 91125, USA

${ }^{2}$ Louisiana State University, Baton Rouge, LA 70803, USA

${ }^{3}$ Università di Salerno, Fisciano, I-84084 Salerno, Italy

${ }^{4}$ INFN, Sezione di Napoli, Complesso Universitario di Monte S.Angelo, I-80126 Napoli, Italy

${ }^{5}$ OzGrav, School of Physics \& Astronomy, Monash University, Clayton 3800, Victoria, Australia ${ }^{6}$ LIGO Livingston Observatory, Livingston, LA 70754, USA

${ }^{7}$ Laboratoire d'Annecy de Physique des Particules (LAPP), Univ. Grenoble Alpes, Université Savoie Mont Blanc, CNRS/IN2P3, F-74941 Annecy, France

${ }^{8}$ University of Sannio at Benevento, I-82100 Benevento, Italy and INFN, Sezione di Napoli, I-80100 Napoli, Italy

${ }^{9}$ Max Planck Institute for Gravitational Physics (Albert Einstein Institute), D-30167 Hannover, Germany

${ }^{10}$ Leibniz Universität Hannover, D-30167 Hannover, Germany

${ }^{11}$ NCSA, University of Illinois at Urbana-Champaign, Urbana, IL 61801, USA

${ }^{12}$ University of Cambridge, Cambridge, CB2 1TN, UK

${ }^{13}$ Nikhef, Science Park 105, 1098 XG Amsterdam, The Netherlands

${ }^{14}$ LIGO, Massachusetts Institute of Technology, Cambridge, MA 02139, USA

15 Instituto Nacional de Pesquisas Espaciais, 12227-010 São José dos Campos, São Paulo, Brazil

${ }^{16}$ Gran Sasso Science Institute (GSSI), I-67100 L'Aquila, Italy

${ }^{17}$ INFN, Laboratori Nazionali del Gran Sasso, I-67100 Assergi, Italy

${ }^{18}$ Inter-University Centre for Astronomy and Astrophysics, Pune 411007, India

${ }^{19}$ International Centre for Theoretical Sciences, Tata Institute of Fundamental Research, Bengaluru 560089, India

${ }^{20}$ University of Wisconsin-Milwaukee, Milwaukee, WI 53201, USA

${ }^{21}$ Università di Pisa, I-56127 Pisa, Italy

22 INFN, Sezione di Pisa, I-56127 Pisa, Italy

${ }^{23}$ Departamento de Astronomía y Astrofísica, Universitat de València, E-46100 Burjassot, València, Spain

${ }^{24}$ OzGrav, Australian National University, Canberra, Australian Capital Territory 0200, Australia

${ }^{25}$ Laboratoire des Matériaux Avancés (LMA), CNRS/IN2P3, F-69622 Villeurbanne, France

${ }^{26}$ SUPA, University of Strathclyde, Glasgow, G1 1XQ, UK

${ }^{27}$ LAL, Univ. Paris-Sud, CNRS/IN2P3, Université Paris-Saclay, F-91898 Orsay, France

${ }^{28}$ California State University Fullerton, Fullerton, CA 92831, USA

${ }^{29}$ APC, AstroParticule et Cosmologie, Université Paris Diderot, CNRS/IN2P3, CEA/Irfu, Observatoire de Paris, Sorbonne Paris Cité, F-75205 Paris Cedex 13, France

${ }^{30}$ European Gravitational Observatory (EGO), I-56021 Cascina, Pisa, Italy

${ }_{31}$ Università di Roma Tor Vergata, I-00133 Roma, Italy

${ }^{32}$ INFN, Sezione di Roma Tor Vergata, I-00133 Roma, Italy

${ }^{33}$ Universität Hamburg, D-22761 Hamburg, Germany

${ }^{34}$ INFN, Sezione di Roma, I-00185 Roma, Italy 


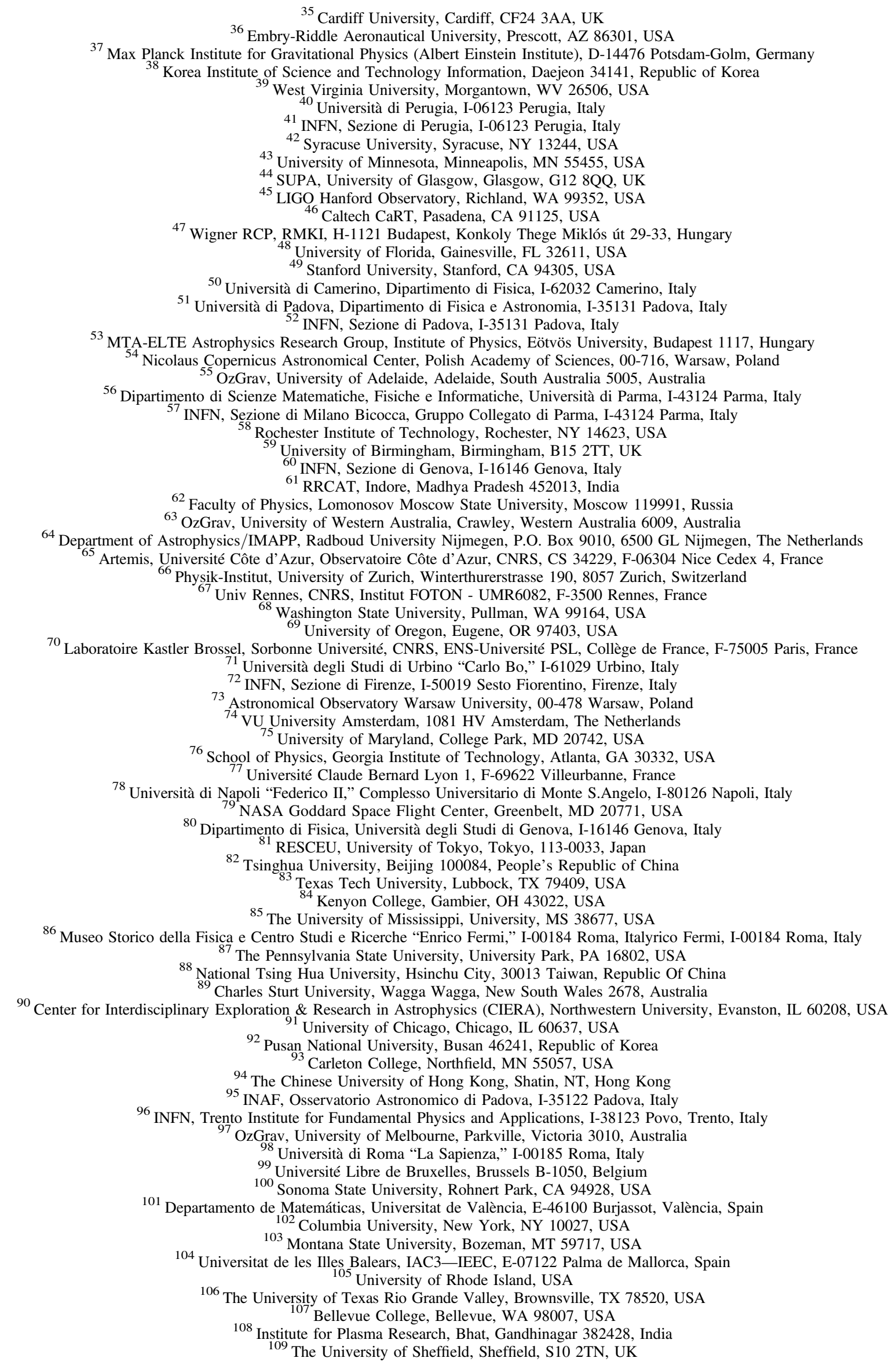




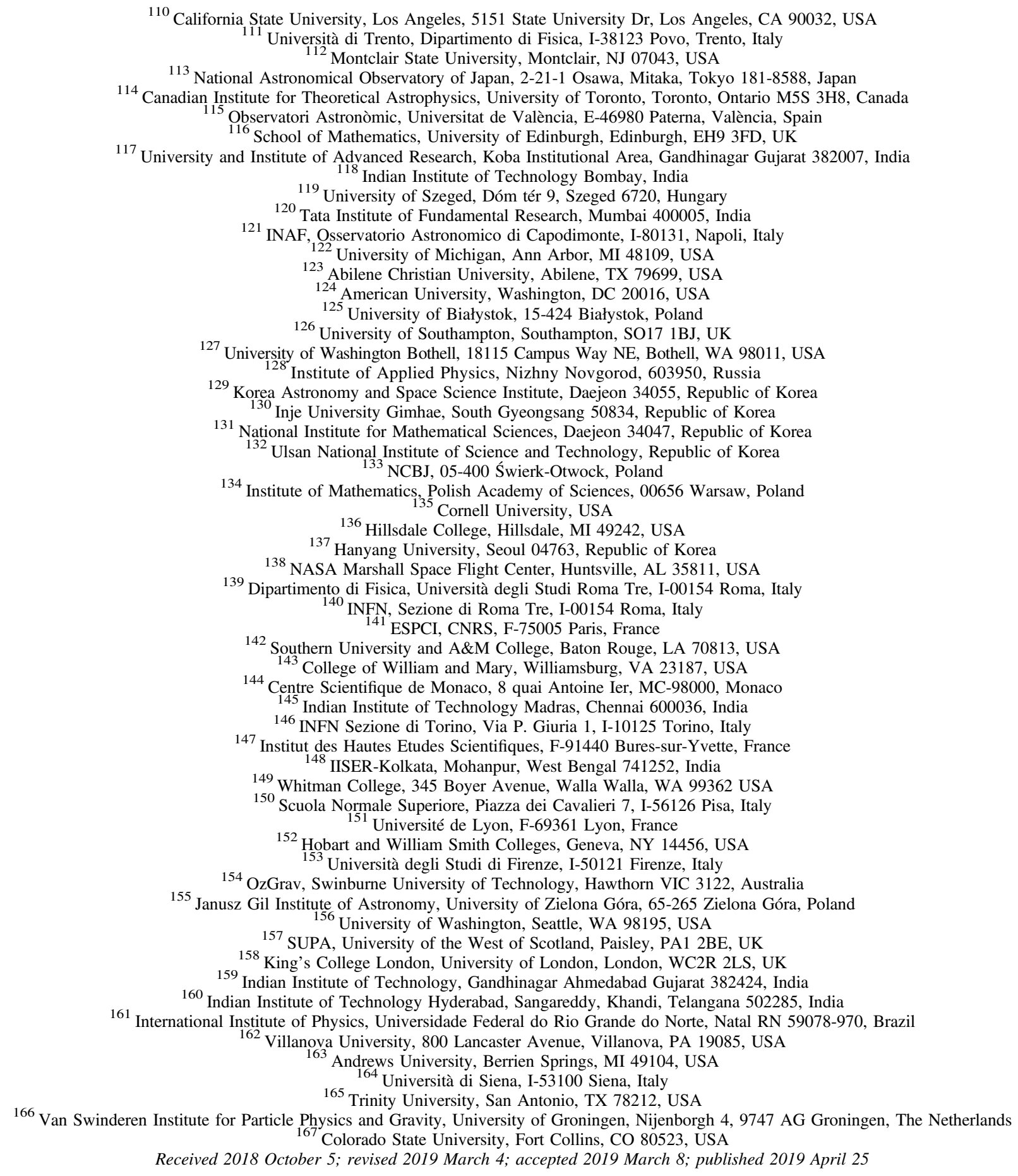

\begin{abstract}
One unanswered question about the binary neutron star coalescence GW170817 is the nature of its post-merger remnant. A previous search for post-merger gravitational waves targeted high-frequency signals from a possible neutron star remnant with a maximum signal duration of $500 \mathrm{~s}$. Here, we revisit the neutron star remnant scenario and focus on longer signal durations, up until the end of the second Advanced LIGO-Virgo observing run, which was 8.5 days after the coalescence of GW170817. The main physical scenario for this emission is the power-law spindown of a massive magnetar-like remnant. We use four independent search algorithms with varying degrees of restrictiveness on the signal waveform and different ways of dealing with noise artefacts. In agreement with theoretical estimates, we find no significant signal candidates. Through simulated signals, we quantify that with the current detector sensitivity, nowhere in the studied parameter space are we sensitive to a signal from more than
\end{abstract}

168 Deceased, 2018 February.
169 Deceased, 2017 November. 
$1 \mathrm{Mpc}$ away, compared to the actual distance of $40 \mathrm{Mpc}$. However, this study serves as a prototype for post-merger analyses in future observing runs with expected higher sensitivity.

Key words: gravitational waves - methods: data analysis - stars: neutron

Supporting material: machine-readable tables

\section{Introduction}

The binary neutron star (BNS) observation GW170817 (Abbott et al. 2017d) was the first multimessenger astronomy event to be jointly detected in gravitational waves (GWs) and at many electromagnetic (EM) wavelengths (Abbott et al. 2017e). It originated remarkably close to Earth, with a distance of $38_{-18}^{+8} \mathrm{Mpc}^{170}$ as measured by the LIGO and Virgo GW detectors (Aasi et al. 2015a; Acernese et al. 2015) alone and consistent EM distance estimates for the host galaxy NGC 4993 (Sakai et al. 2000; Freedman et al. 2001; Hjorth et al. 2017; Lee et al. 2018).

A BNS merger is expected to leave behind a remnant compact object, either a light stellar-mass black hole or a heavy neutron star (NS), which can emit a variety of post-merger GW signals. Although these are more difficult to detect than the premerger inspiral signal, the nearby origin of GW170817 has generated interest in the search for a post-merger signal. Identifying the nature of the remnant would be highly valuable for improving, among other things, constraints on the nuclear equation of state (EoS) (Bauswein et al. 2017; Margalit \& Metzger 2017; Radice et al. 2018; Rezzolla et al. 2018) over those obtained from the inspiral alone (e.g., Abbott et al. 2017d, 2018c, 2019).

Abbott et al. (2017g) presented the first model-agnostic search for short $(\lesssim 1 \mathrm{~s})$ and intermediate-duration $(\lesssim 500 \mathrm{~s}) \mathrm{GW}$ signals. However, no signal candidates were found. The search sensitivity was estimated for several GW emission mechanisms, including oscillation modes of a short-lived hypermassive NS, bar-mode instabilities, and rapid spindown powered by magnetic-field induced ellipticities. For all of these mechanisms, a realistic signal from a NS remnant of GW170817 could only have been detected with at least an order of magnitude increase in detector strain sensitivity. A seconds-long postmerger signal candidate was reported by van Putten \& Della Valle (2018), with an estimated GW energy lower than the sensitivity estimates of Abbott et al. (2017g).

An additional analysis in Abbott et al. (2019) used a Bayesian wavelet-based method to put upper limits on the energy and strain spectral densities over $1 \mathrm{~s}$ of data around the coalescence. These strain upper limits are 3-10 times above the numerical relativity expectations for post-merger emission from a hypermassive NS at $40 \mathrm{Mpc}$.

In this paper, we focus on a long-lived NS remnant and we cover many possible signal durations, which, at the long end, are limited by the end of the second observing run $(\mathrm{O} 2)$ on 2017 August 26. This gives a total dataset that spans 8.5 days from merger. The shortest signal durations that we cover are $\sim$ hundreds of seconds after merger, so that the new search presented here only partially overlaps with the intermediateduration search from Abbott et al. (2017g). We assume the sky location of the EM counterpart (Abbott et al. 2017e; Coulter et al. 2017).

\footnotetext{
${ }^{170}$ Updated distance estimate corresponding to Figure 3 of Abbott et al. (2019), where the sky location of the counterpart is not assumed, hence differing slightly from the one quoted in the text for fixed-location runs.
}

From considerations of realistic remnant NS properties, which are detailed in Section 2, we do not expect to make a detection with this search. Instead, the goal-as before in Abbott et al. (2017g) - is mainly to make sure that no unexpected signal is missed in the longer-duration part of the parameter space. This study also serves as a rehearsal for future post-merger searches with improved detectors. Hence, we use the following four search methods, with varying restrictiveness on the signal shape and different ways of dealing with noise artefacts: two generic unmodeled algorithms and two that use templates based on a power-law spindown waveform model.

The Stochastic Transient Analysis Multidetector Pipeline (STAMP, Thrane et al. 2011) is an unmodeled method that uses cross-power spectrograms. This method has already been used for the intermediate-duration analysis in Abbott et al. (2017g), but is employed here in a different configuration that is optimized for much longer signal lengths.

The other three algorithms are derived from methods originally developed to search for continuous waves (CWs): persistent, nearly-monochromatic GW signals from older NSs. (For reviews, see Prix 2009; Riles 2017.) Some CW searches have targeted relatively young NSs (Aasi et al. 2015b; Sun et al. 2016; Zhu et al. 2016), and adaptations of $\mathrm{CW}$ search methods to long-duration transient signals have previously been suggested (Prix et al. 2011; Keitel 2016). However, the present search is the first time that any $\mathrm{CW}$ algorithms have been modified in practice (on real data) to deal with transients of rapid frequency evolution.

Specifically, these three are: Hidden Markov Model (HMM) tracking (Suvorova et al. 2016; Sun et al. 2018), which is a template-free algorithm that has previously been used to search for CWs from the binary Scorpius X-1 (Abbott et al. 2017f); and two new model-dependent methods-Adaptive Transient Hough (ATrHough, Oliver et al. 2019) and Generalized FrequencyHough (FreqHough, Miller et al. 2018)—, which are based on algorithms (Krishnan et al. 2004; Palomba et al. 2005; Sintes \& Krishnan 2007; Antonucci et al. 2008; Aasi et al. 2014; Astone et al. 2014) that have previously been used in CW all-sky searches (e.g., most recently in Abbott et al. 2017a, 2018b).

After discussing the astrophysical motivation and context for this search in Section 2, we present the analyzed dataset in Section 3. We then describe the four search methods in Section 4 and discuss the combined search results in Section 5. Finally, we conclude with remarks on future applications in Section 6. Additional results and details on the search methods are given in the appendices.

\section{Astrophysical Background and Waveform Model}

The probability for a long-lived NS remnant after a BNS merger depends on the progenitor properties and on the nuclear EoS (Baiotti \& Rezzolla 2017; Piro et al. 2017). Using the progenitor masses and spins as measured from the inspiral 
(Abbott et al. 2017d, 2019), for many EoS the preferred scenarios are the prompt collapse to a black hole or the formation of a hypermassive NS whose mass cannot be supported by uniform rotation and thus collapses in $\lesssim 1 \mathrm{~s}$ (Abbott et al. 2017c). However, a supramassive NS-less massive, but above the maximum mass of a nonrotating NS and stable for up to $\sim 10^{4} \mathrm{~s}$ (Ravi \& Lasky 2014)_or even a long-time stable NS could also be consistent with some physically-motivated EoS which allow for high maximum masses.

From the EM observational side, circumstantial evidence points toward a short-lived hypermassive NS (Granot et al. 2017, 2018; Kasen et al. 2017; Matsumoto et al. 2018; Pooley et al. 2018); though several authors (Ai et al. 2018; Geng et al. 2018; Li et al. 2018; Yu et al. 2018) have considered continued energy injection from a long-lived remnant NS. Given this inconclusive observational situation, we agnostically consider the possibility of GW emission from a long-lived remnant NS and seek here to constrain it from the LIGO data.

In two of our search methods, and to estimate search sensitivities with simulations, we use a waveform model (Lasky et al. 2017; Sarin et al. 2018) that originates from the general torque equation for the spindown of a rotating NS:

$$
\dot{\Omega}=-k \Omega^{n} .
$$

Here, $\Omega=2 \pi f$ and $\dot{\Omega}$ are the star's angular frequency and its time derivative, respectively, and $n$ is the braking index. A value of $n \leqslant 3$ corresponds to spindown predominantly through magnetic dipole radiation and $n=5$ to pure $\mathrm{GW}$ emission (Shapiro \& Teukolsky 1983). A braking index of $n=7$ is conventionally associated with spindown through unstable $r$-modes (e.g., Owen et al. 1998), although the true value can be less for different saturation mechanisms (Alford \& Schwenzer 2015, 2014). The value of $k$ also depends on these mechanisms; together with the starting frequency $\Omega_{0}$, it defines a spin-down timescale parameter

$$
\tau=-\frac{\Omega_{0}^{1-n}}{k(1-n)}
$$

Integrating Equation (1) and solving for the GW frequency gives the GW frequency evolution

$$
f_{\mathrm{gw}}(t)=f_{\mathrm{gw} 0}\left(1+\frac{t}{\tau}\right)^{1 /(1-n)}
$$

where $f_{\mathrm{gw}}=2 f, f_{\mathrm{gw} 0}$ is the initial frequency at a starting time $t_{\text {start }}$ (e.g., coalescence time $t_{\mathrm{c}}$ of the BNS merger), and $t$ is measured relative to $t_{\text {start }}$.

The dimensionless GW strain amplitude for a non-axisymmetric rotating body following Equation (3) is given by

$$
h_{0}(t)=\frac{4 \pi^{2} G I_{z z}}{c^{4}} \frac{\epsilon}{d} f_{\mathrm{gw} 0}^{2}\left(1+\frac{t}{\tau}\right)^{2 /(1-n)} .
$$

Here, $I_{z z}$ is the principal moment of inertia, $\epsilon$ is the ellipticity of the rotating body, $d$ is the distance to the source, $G$ is the gravitational constant, and $c$ is the speed of light. This model assumes that $n, \epsilon$ and $I_{z z}$ are constant throughout the spin-down phase, while in reality the spindown could be e.g., GWdominated at early times and then transition into EM dominance, and $I_{z z}$ can decrease with $\Omega$.

Our set of pipelines also allows for the power-law spindown model to be valid for only part of the observation time. To accommodate the possibility that the newborn NS has not immediately settled into a state that obeys the powerlaw model, the FreqHough analysis starts a few hours after the merger (at $t_{\mathrm{c}} \approx 1187008882.443$ in GPS seconds, with the offset $\Delta t$ varying across parameter space as described later), making no assumption about the earlier NS evolution. This provides complementary constraints to the other analyses. The unmodeled STAMP search is also sensitive to signals starting at either $t_{\mathrm{c}}$ or at any later time because it does not impose a fixed starting time for any time-frequency tracks. Moreover, neither STAMP nor HMM impose the specific waveform model for their initial candidate selection.

The theoretical detectability of newborn NSs evolving according to the spin-down model, Equation (3), has been explored previously, beginning with simple matched-filter estimates (Palomba 2001; Dall'Osso et al. 2009). More recent estimates also consider the limitations of practical searches in the context of magnetars born following core-collapse supernovae (Dall'Osso et al. 2018) and long-lived postmerger remnants (Dall'Osso et al. 2015, 2018; Sarin et al. 2018), finding qualitatively similar results. With Advanced LIGO (aLIGO) at design sensitivity (Abbott et al. 2018d) and an optimal matched-filter analysis, at $d=40 \mathrm{Mpc}$ an ellipticity $\epsilon \sim 10^{-2}$ and timescale $\tau \gtrsim 10^{4} \mathrm{~s}$ would be required. However, such a large $\epsilon$ and long $\tau$ would imply more energy emitted than is available from the remnant's initial rotation. Considering actual data analysis pipelines applied to real detector data (at $\mathrm{O} 2$ sensitivity), a detectable signal only seems possible for extremely large $\epsilon \geqslant 0.1$ and short $\tau$ due to the energy budget constraint (Sarin et al. 2018). However, these ellipticities are physically unlikely (JohnsonMcDaniel \& Owen 2013) and would require internal magnetic fields greater than $\sim 10^{17} \mathrm{G}$ (e.g., Cutler 2002), which might be intrinsically unstable (Reisenegger 2009) and for which very rapid EM-dominated spindown would be expected.

For r-modes, the GW strain follows a different relation than Equation (4) (Owen et al. 1998), but the physically relevant parameter (the saturation amplitude) is also expected to be small (Arras et al. 2003; Bondarescu et al. 2009). These r-modes could be an important emission channel, especially at high frequencies, and the search presented in this paper also covers braking indices up to $n=7$. However, for the sake of simplicity, the sensitivity estimates presented in Section 5 will be for $n=5$ only.

Nevertheless, with this first search for long-duration postmerger signals, we demonstrate that the available analysis methods can comprehensively cover the relevant parameter space, and thus will be ready once detector sensitivity has improved or in the case of a fortunate, very nearby BNS event. 

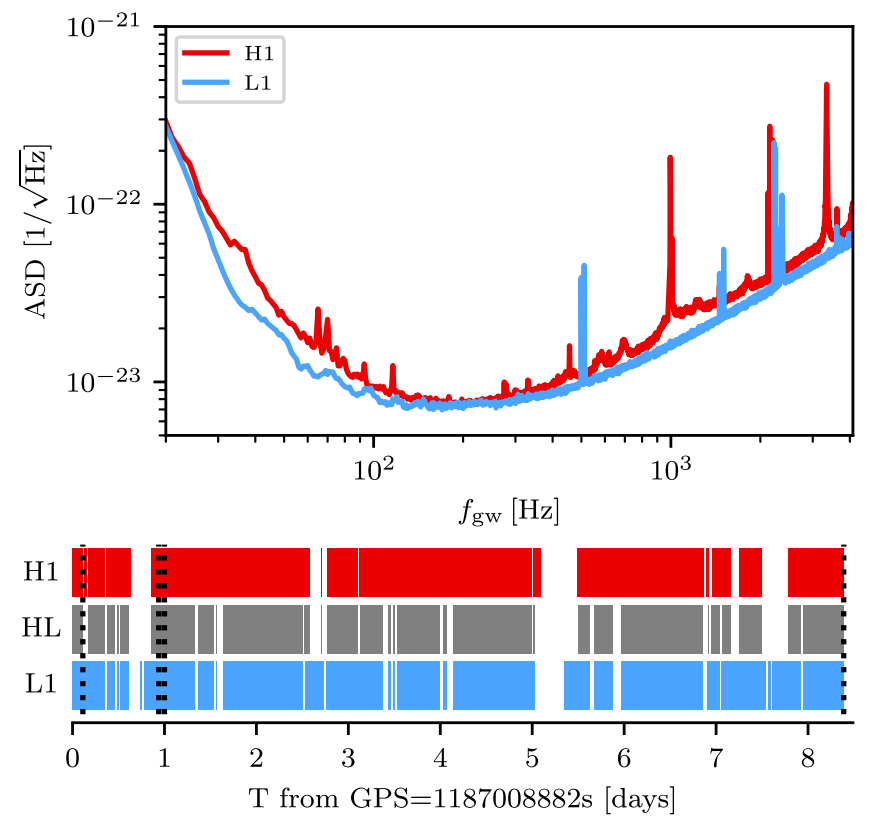

Figure 1. Top panel: noise strain amplitude spectral density (ASD) curves of LIGO Hanford (H1) and Livingston (L1) on 2018 August 17. (Averaged over 1800 s stretches including GW170817.) Lower panel: analyzable science mode data segments for the remaining O2 run after the GW170817 event. Verticaldotted lines mark the analysis end times, from left to right, for HMM, FreqHough, ATrHough and STAMP.

\section{Detectors and Dataset}

In this analysis, we use data from the two aLIGO detectors in Hanford, Washington (H1) and Livingston, Louisiana (L1). No data from Virgo (Acernese et al. 2015) or GEO600 (Dooley et al. 2016) was used because of their lower sensitivity. ${ }^{171}$ Three of the pipelines use data up to $2 \mathrm{kHz}$, while STAMP also uses data up to $4 \mathrm{kHz}$. Both detectors in their $\mathrm{O} 2$ configuration had their best sensitivity in the $100-200 \mathrm{~Hz}$ range, with significantly less sensitivity in the $\mathrm{kHz}$ range (e.g., a factor $\sim 4$ worse in strain at $2 \mathrm{kHz}$ ) - see Figure 1. For the lower analysis cutoff of each pipeline, see Section 4.

Starting from a rounded GW170817 coalescence time of $t_{\mathrm{c}} \approx 1187,008,882 \mathrm{~s}$, the HMM pipeline uses $9688 \mathrm{~s}$ of data (until the first gap in $\mathrm{H} 1$ data), ATrHough uses 1 day of data after $t_{\mathrm{c}}$, FreqHough analyzes from 1 to about $18 \mathrm{hr}$ after $t_{\mathrm{c}}$ in different configurations, and STAMP analyzes the whole 8.5 days of data until the end of $\mathrm{O} 2$ on 2017 August 26. The duty cycle of both detectors $(\mathrm{H} 1, \mathrm{~L} 1)$ was $100 \%$ during the first $9688 \mathrm{~s}$ after the merger, $(70 \%, 78 \%)$ for the first day $(62 \%$ in coincidence), and $(83 \%, 85 \%)$ for the full dataset $(75 \%$ in coincidence). The analyzed data segments are also illustrated in Figure 1. STAMP processes the $h(t)$ strain data into crosspower time-frequency maps (see Section 4.1 for details), while the basic analysis units of the other pipelines are Short Fourier Transforms (SFTs) of 1-8 s duration.

Several known noise sources have been subtracted from the strain data using a new automated procedure (Davis et al. 2019) that has been applied to the full O2 dataset, which processes a much larger amount of time than the cleaning method (Driggers et al. 2019) that was used for the shorter

\footnotetext{
${ }^{171}$ For example, at $500 \mathrm{~Hz}$ the noise strain amplitude spectral density was about a factor of $\sim 5$ for Virgo and $\sim 20$ for GEO600 worse than for L1 in late $\mathrm{O} 2$.
}

datasets analyzed in previous GW170817 publications. Calibration uncertainties (Cahillane et al. 2017) for this dataset are estimated as below $4.3 \%$ in amplitude and $2^{\circ} .3$ in phase for $20-2000 \mathrm{~Hz}$, and $4.5 \%$ in amplitude and 3.8 in phase for $2-4 \mathrm{kHz}$ - which are tighter than for the initial calibration version used in Abbott et al. (2017g). These uncertainties are not explicitly propagated into the sensitivity estimates presented in this paper because they are smaller than other uncertainty contributions and the degeneracies in amplitude parameters.

\section{Search Methods and Configurations}

In this section, we briefly describe the four search methods, first the unmodeled STAMP and HMM pipelines and then the two Hough pipelines tailored to the power-law spindown model. Additional details can be found in Appendix A.

Each analysis uses the known sky location of the counterpart near R.A. $=13.1634 \mathrm{hr}$, decl. $=-23.3815$ (Abbott et al. 2017e; Coulter et al. 2017), but makes different choices for the analyzed data span. The recovery efficiency of each algorithm is studied with simulated signals under the waveform model from Section 2, as described in Section 5 and Appendix B.

A summary of configurations for all four pipelines, both for the main search and the sensitivity estimation simulations, is given in Table 1.

\subsection{STAMP}

STAMP (Thrane et al. 2011) is an unmodeled search pipeline that is designed to detect gravitational wave transients. Its basic unit is a spectrogram made from cross-correlated data between two detectors. Narrowband transient gravitational waves produce tracks of excess power within these spectrograms and can be detected by pattern-recognition algorithms. Each spectrogram pixel is normalized with the noise to obtain a signal-to-noise ratio $(\mathrm{S} / \mathrm{N})$ for each pixel.

STAMP was used in the first GW170817 post-merger search (Abbott et al. 2017g) in a configuration with $500 \mathrm{~s}$ long spectrograms. To increase sensitivity to longer GW signals, here we use spectrogram maps of 15,000 s length. The search is split into two frequency bands from 30 to 2000 and 2000 to $4000 \mathrm{~Hz}$. The former uses pixels of $100 \mathrm{~s} \times 1 \mathrm{~Hz}$, while the latter uses shorter-duration pixels of $50 \mathrm{~s} \times 1 \mathrm{~Hz}$ to limit $\mathrm{S} / \mathrm{N}$ loss due to the Earth's rotation changing the phase difference between detectors.

We then use Stochtrack (Thrane \& Coughlin 2013), which is a seedless clustering algorithm, to identify significant clusters of pixels within these maps. The algorithm uses one million quadratic Bézier curves as templates for each map and the loudest cluster is picked for each map. More details about the pixel size choice, the detection statistic and the search results are given in Appendix A.1.

The on-source data window is from just after the time of the merger to the end of O2 (1187008942-1187733618). To measure the background and estimate the significance of the clusters that we have found, we run the algorithm on timeshifted data from June 24th to just before the merger.

\subsection{HMM Tracking}

Hidden Markov model (HMM) tracking provides a computationally efficient strategy to detect and estimate a 
Table 1

Configurations of the Four Analysis Pipelines Used in This Paper

\begin{tabular}{|c|c|c|c|c|}
\hline & STAMP & HMM & ATrHough & FreqHough \\
\hline Search start $^{\mathrm{a}}$ & $t_{\mathrm{c}}$ & $t_{\mathrm{c}}$ & $t_{\mathrm{c}}$ & $t_{\mathrm{c}}+(1-7) \mathrm{hr}^{\mathrm{b}}$ \\
\hline Search duration (hr) & $201.3^{\mathrm{c}}$ & 2.7 & 24 & $2-18^{\mathrm{b}}$ \\
\hline$f_{\mathrm{gw}}$ data range $(\mathrm{Hz})$ & $30-4000^{c}$ & $100-2000$ & $187-2000$ & $50-2000$ \\
\hline$n$ coverage & unmodeled & unmodeled & $2.5-7.0$ & $2.5-7.0$ \\
\hline$f_{\text {start }}$ coverage $(\mathrm{Hz})^{\mathrm{d}}$ & unmodeled & unmodeled & $500-2000$ & $500-2000$ \\
\hline$\tau$ coverage $(\mathrm{s})$ & unmodeled & unmodeled & $10^{2}-10^{5}$ & $10-10^{5}$ \\
\hline \multicolumn{5}{|c|}{ injection set for sensitivity estimation $^{\mathrm{e}}$} \\
\hline Signal start $\mathrm{a}^{\mathrm{a}}$ & random & $t_{\mathrm{c}}$ & $t_{\mathrm{c}}$ & $t_{\mathrm{c}}+[1,2,5] \mathrm{hr}^{\mathrm{b}}$ \\
\hline$n$ coverage & 5.0 & $2.5-7.0$ & 5.0 & 5.0 \\
\hline$f_{\text {start }}$ coverage $(\mathrm{Hz})^{\mathrm{d}}$ & $500-3000$ & $500-2000$ & $550-2000$ & 390-2000 \\
\hline$\tau$ coverage (s) & $10^{2}-10^{4}$ & $10^{2}-10^{4}$ & $6 \times 10^{2}-3 \times 10^{4}$ & $4 \times 10^{2}-2 \times 10^{4}$ \\
\hline Inclination $\cos \iota$ & $0.0,1.0$ & random & $0.0,1.0$ & random \\
\hline
\end{tabular}

Notes.

${ }^{\text {a }}$ Coalescence time $t_{\mathrm{c}} \approx 1187,008,882$ rounded to integer GPS seconds.

${ }^{\mathrm{b}}$ FreqHough search start and duration vary across parameter space.

${ }^{\mathrm{c}}$ In separate maps of $15,000 \mathrm{~s}$ length and $20-2000$ and $2000-4000 \mathrm{~Hz}$ configurations.

$\mathrm{d} f_{\text {start }}=f_{\mathrm{gw}}(t=0)$ for HMM and ATrHough; $f_{\text {start }}=f_{\mathrm{gw}}(t=\Delta t)$ for STAMP and FreqHough.

${ }^{\mathrm{e}}$ Discrete sets of injections within these ranges; not all combinations used. See Section 5 and the per-pipeline tables in the appendix for details.

quasimonochromatic GW signal with unknown frequency evolution and stochastic timing noise (Suvorova et al. 2016; Sun et al. 2018). It was applied to data from the first aLIGO observing run to search for CWs from the low-mass X-ray binary Scorpius X-1 (Abbott et al. 2017f). The revision of the algorithm in Sun et al. (2018) is also well-suited to searching for a long-transient signal from a BNS merger remnant, if the spin-down timescale is in the range $10^{2} \mathrm{~s} \lesssim \tau \lesssim 10^{4} \mathrm{~s}$.

A HMM is an automaton that is based on a Markov chain (a stochastic process transitioning between discrete states at discrete times), which is composed of a hidden (unmeasurable) state variable and a measurement variable. A HMM is memoryless; i.e., the hidden state at time $t_{n+1}$ only depends on the state at time $t_{n}$, with a certain transition probability. The most probable sequence of hidden states given the observations is computed by the classic Viterbi algorithm (Viterbi 1967). Further details on the probabilistic model can be found in Appendix A.2.

In this analysis, we track the $\mathrm{GW}$ signal frequency as the hidden variable, whose discrete states are mapped one-to-one to the frequency bins in the output of a frequency-domain estimator computed over an interval of length $T_{\text {drift }}$. We aim to search for signals with $10^{2} \mathrm{~s} \lesssim \tau \lesssim 10^{4} \mathrm{~s}$, such that the first time derivative $\dot{f}_{\mathrm{gw}}$ of the signal frequency $f_{\mathrm{gw}}$ satisfies $\dot{f}_{\mathrm{gw}} \approx$ $f_{\mathrm{gw}} / \tau \lesssim 1 \mathrm{~Hz} \mathrm{~s}^{-1}$, given $T_{\text {drift }}=1 \mathrm{~s}$ and a frequency bin width of $\Delta f=1 \mathrm{~Hz}$. The motion of the Earth with respect to the solar system barycenter (SSB) can be neglected during a $T_{\text {drift }}$ interval. Hence, we use a running-mean normalized power in SFTs with length $T_{\mathrm{SFT}}=T_{\mathrm{drift}}=1 \mathrm{~s}$ as the estimator to calculate the HMM emission probability.

We analyze $9688 \mathrm{~s}$ of data (GPS times 11870088821187018570 ) in a $100-2000 \mathrm{~Hz}$ frequency band with multiple configurations optimized for different $\tau$. We do not analyze longer data stretches because: (i) several intervals in the data after GPS time 1187018570 are not in analyzable science mode, and (ii) signals with $10^{2} \mathrm{~s} \lesssim \tau \lesssim 10^{4} \mathrm{~s}$ drop below the algorithm's sensitivity limit after $\sim 10^{4} \mathrm{~s}$. Observing for longer merely accumulates noise without improving $\mathrm{S} / \mathrm{N}$. The 9688 SFTs are Hann-windowed. The detection statistic $\mathcal{P}$ is defined in Equation (17). The methodology and analysis is fully described in Sun \& Melatos (2018).

\subsection{Adaptive Transient Hough}

The Adaptive Transient Hough search method is described in detail in Oliver et al. (2019). It follows a semi-coherent strategy similar to the SkyHough (Krishnan et al. 2004; Sintes \& Krishnan 2007; Aasi et al. 2014) all-sky CW searches, but adapted to rapid-spindown transient signals.

We start from data in the form of Hann-windowed SFTs with lengths of $[1,2,4,6,8] \mathrm{s}$, covering one day after merger (GPS times 1187008882-1187095282). These are digitized by setting a threshold of 1.6 on their normalized power, as first derived by Krishnan et al. (2004), replacing each SFT by a collection of zeros and ones called a peak-gram. For each point in parameter space, the Hough number count is the weighted sum of the peak-grams across a template track accounting for Doppler shift and the spindown of the source. The use of weights minimizes the influence of time-varying detector antenna patterns and noise levels (Sintes \& Krishnan 2007). For this post-merger search, it also accounts for the amplitude modulation related to the transient nature of the signal.

The search parameter space for the model from Section 2 covers a band of $500-2000 \mathrm{~Hz}$ in starting frequencies $f_{\mathrm{gw} 0}$, braking indices of $2.5 \leqslant n \leqslant 7$ and spindown timescales of $10^{2} \leqslant \tau \leqslant 10^{5} \mathrm{~s}$. The search runs over 16,042 subgroups, each containing a range of $150 \mathrm{~Hz}$ in $f_{0}, 0.25$ in $n$ and $1000 \mathrm{~s}$ in $\tau$. Each subgroup is analyzed with the longest possible SFTs according to the criterion (Oliver et al. 2019)

$$
T_{\mathrm{SFT}} \leqslant \frac{\sqrt{(n-1) \tau}}{\sqrt{f_{\mathrm{gw}}}}
$$

and for each template the observation time is selected as $T_{\text {obs }}=\min (4 \tau, 24 \mathrm{hr})$. Over the whole template bank, the search uses data from 187 to $2000 \mathrm{~Hz}$.

Each template is ranked based on the deviation of its weighted number count from the theoretical expectation for Gaussian noise 
(the critical ratio) as described in Appendix A.3. The detection threshold corresponds to a two-detector $5 \sigma$ false-alarm probability for the entire template bank. A per-detector critical ratio threshold was also set to check the consistency of a signal between $\mathrm{H} 1$ and $\mathrm{L} 1$.

\subsection{Generalized FrequencyHough}

The FrequencyHough is a pattern-recognition technique that was originally developed to search for CWs by mapping points in time-frequency space of the detector to lines in frequencyspindown space (Antonucci et al. 2008; Astone et al. 2014). This only works if the signal frequency varies in time very slowly. Miller et al. (2018) have generalized the FrequencyHough for post-merger signals, where we expect much higher spindowns.

The search starts at a time offset $\Delta t=t_{\text {start }}-t_{\mathrm{c}}$ after coalescence time $t_{\mathrm{c}}$, so that the waveform model is interpreted with starting frequency $f_{\text {start }}=f_{\mathrm{gw}}(t=\Delta t)$ taking the place of $f_{\mathrm{gw} 0}$ in Equation (3). In this way, assuming that the NS has already spun down before $t_{\text {start }}$ following some arbitrary track, we would be able to probe higher initial frequencies and spindowns through a less challenging parameter space during the search window. Furthermore, the source parameters $\left(n, f_{\text {start }}, \tau\right)$ are transformed to new coordinates, such that in the new space the behavior of the signal is linear. See Appendix A.4 for the transformation relations.

We search across the parameter space with a fine, nonuniform grid: for each braking index $n$, we do a Hough transform and then record the most significant candidates over the parameter range of the resulting map. This is done separately on the data from each detector. We then check the candidates for coincidence between detectors according to their Euclidean distance in parameter space.

The search is run in three configurations using varying $T_{\mathrm{SFT}}=2,4,8 \mathrm{~s}$, covering different observing times, starting $\Delta t=1-7 \mathrm{hr}$ after merger. It covers $n=[2.5,7], f_{\text {start }}=$ $[500,2000] \mathrm{Hz}$ and $\tau=\left[10,10^{5}\right] \mathrm{s}$, analyzing detector data from 50 to $2000 \mathrm{~Hz}$.

Candidates are also ranked by critical ratio (deviation from the theoretical expectation for Gaussian noise) in this analysis. Most can be vetoed by the coincidence step or by considering detector noise properties. A follow-up procedure for surviving candidates is also described in Appendix A.4.

\section{Search Results and Sensitivity Estimates}

\subsection{Absence of Significant Candidates}

All of the four search methods either found no significant candidates in the aLIGO data after GW170817; or those that were found, were clearly vetoed as instrumental artifacts.

For the unmodeled STAMP search, the loudest triggers in the low- and high-frequency bands have S/Ns of 3.18 and 3.07, respectively. The time-shifted backgrounds only just start to drop off near these S/Ns, so that they correspond to falsealarm probabilities $p_{\mathrm{FA}}$ of 0.81 and 0.80 , which are completely consistent with noise. For reference, $p_{\mathrm{FA}}=0.05$ would only have been reached for $S / N$ s of $\gtrsim 4.9$ and $\gtrsim 3.5$ for these lowand high-frequency background distributions, respectively. (See Figure 4 in Appendix A.1.)

For HMM, the loudest trigger has a detection statistic $\mathcal{P}=2.6749$ (as defined in Equation (17)), which corresponds to a false-alarm probability of 0.01 , right below the threshold set beforehand as significant enough for further study. The trigger is found with observing time $T_{\mathrm{obs}}=200 \mathrm{~s}$ starting from $t=t_{\mathrm{c}}$. Monte-Carlo simulations show that for the signals that this setup is sensitive to, a higher $\mathcal{P}$ should be obtained with longer $T_{\text {obs }}$. Follow-up analysis of the trigger with $300 \mathrm{~s} \leqslant$ $T_{\text {obs }} \leqslant 1000 \mathrm{~s}$ confirms that it does not follow this expectation; hence, it is discarded as spurious.

ATrHough found 51 initial candidates over the covered part of $\left(n, f_{\mathrm{gw} 0}, \tau\right)$ parameter space. All of these were excluded with the follow-up procedure described in Appendix A.3 because they are inconsistent with the expected spindown model and are more likely to have been caused by monochromatic detector artifacts (lines) contaminating the search templates.

The FreqHough search returned 521 candidates over the covered part of $\left(n, f_{\text {start }}, \tau\right)$ parameter space. We vetoed 10 of them because they were within frequency bands contaminated with known noise lines (Covas et al. 2018). A total of 510 of the remaining candidates had much higher ( $>4$ times) critical ratios in $\mathrm{H} 1$ than in $\mathrm{L} 1$, which is inconsistent with true astrophysical signals when considering the relative sensitivities, duty factors and antenna patterns. There was one remaining candidate, with a critical ratio of 5.21 in $\mathrm{H} 1$ and 4.88 in L1, which was followed up and excluded with the procedure described in Appendix A.4.

\subsection{Sensitivity Estimates with Simulated Signals}

Starting from this non-detection result, we use simulated signals according to Equation (3) to quantify the sensitivity of each analysis given the dataset around the time of GW170817 and its known sky location. The sets of injected parameters are different for each pipeline, while there are also some differences in procedure: STAMP performs injections on the same data as the main search but with a non-physical time shift between the detectors (as in Abbott et al. 2017g, 2018a); HMM injects signals into the original set of SFTs but with randomly permuted timestamps; and the other two pipelines inject signals into exactly the same data as analyzed in the main search. HMM and ATrHough perform all injections starting at merger time $t_{\mathrm{c}}$, with $f_{\mathrm{gw} 0}$ in Equation (3) interpreted as the frequency at $t_{\mathrm{c}}$, while injections for FreqHough are done at $\Delta t=1,2$ or $5 \mathrm{hr}$ after $t_{\mathrm{c}}$, which are chosen as representative starting times for each search configuration, and $f_{\mathrm{gw} 0}$ is correspondingly set at $t_{\mathrm{c}}+\Delta t$. Similarly, STAMP treats $f_{\mathrm{gw} 0}$ as the starting frequency of each injection, which have $\Delta t$ distributed through the whole search range, yielding a time-averaged sensitivity. In the following, we use $f_{\text {start }}$ to refer to any of these choices.

These differences in injection procedure and the different choices of detection threshold mean that any comparison of the following results does not correspond to a representative evaluation of general pipeline performance but is solely in the interest of estimating how much sensitivity is missing for a GW170817-like post-merger detection that is based on the specific configurations that are used in the present search.

We focus here on results for a braking index of $n=5$, as expected for spin-down dominated by $\mathrm{GW}$ emission from a static quadrupole deformation. The signal amplitude $h_{0}$ (as given in Equation (4)) is degenerate between the ellipticity $\epsilon$, moment of inertia $I_{z z}$ and distance $d$. We choose a fiducial value of $I_{z z}=$ $100 M_{\odot}^{3} G^{2} / c^{4} \approx 4.34 \times 10^{38} \mathrm{~kg} \mathrm{~m}^{2}$, which is consistent with EoS yielding a supramassive or stable remnant: the high mass and assumed rapid rotation can increase the moment of inertia by more than a factor of 3 compared to a nonrotating NS of $1.4 M_{\odot}$. In addition, EoS compatible with the high remnant mass favor larger moments of inertia already at lower mass. For a given set of 

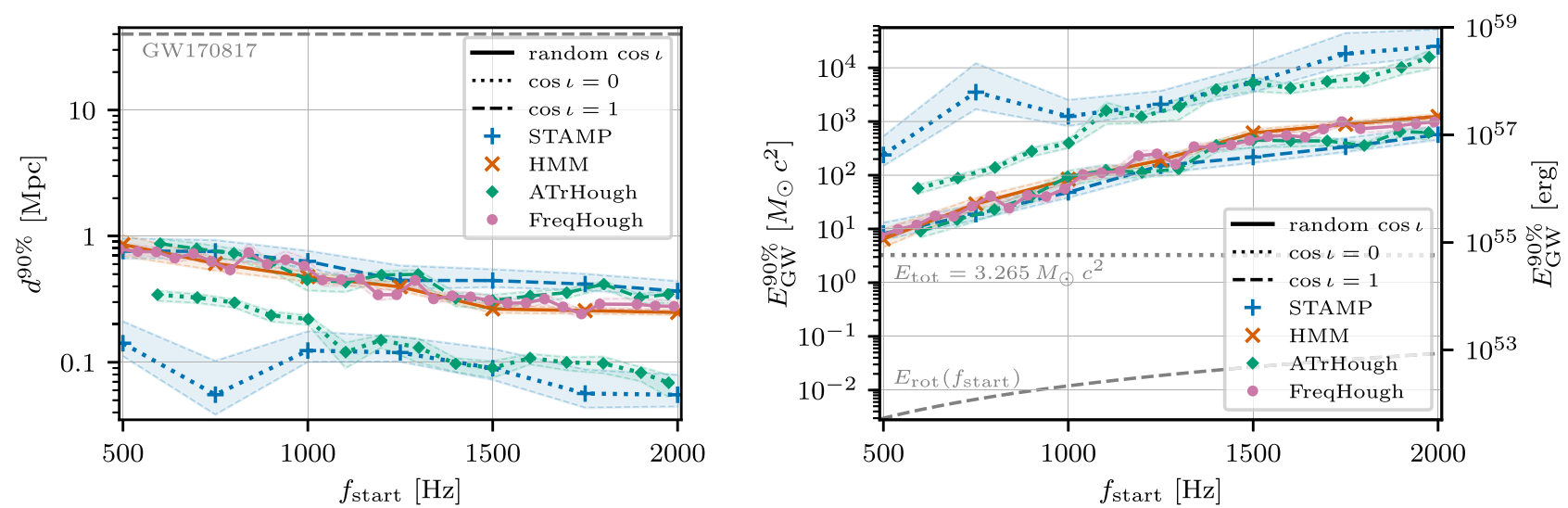

Figure 2. A sample of search sensitivities achieved for the power-law spindown signal model with braking index $n=5$. Results are shown as sensitive distance $d^{90 \%}$ (lefthand panel) for otherwise physical parameters, or as required emitted energy $E_{\mathrm{gw}}^{90 \%}$ at a fixed distance $d=40 \mathrm{Mpc}$ (right-hand panel), both as a function of reference starting frequency $f_{\text {start }}$ used for the injections of each pipeline. $\left(f_{\text {start }}=f_{\mathrm{gw}}(t=\Delta t)\right.$ for STAMP and FreqHough and $f_{\text {start }}=f_{\mathrm{gw} 0}=f_{\mathrm{gw}}(t=0)$ for the others.) See Figure 3 for the parameter ranges covered by each injection set. This figure shows the subset with highest sensitivity for each analysis; this corresponds to the shortest $(\tau=100 \mathrm{~s})$ injections for STAMP and HMM, while for ATrHough and FreqHough $\tau\left(f_{\text {start }}\right)$ is variable, depending on the search coherence length, as also listed in Tables 4 and 5. Note that detection thresholds are also different between pipelines. The NS ellipticity $\epsilon$ is always chosen as the maximum allowed by the energy budget constraint $E_{\mathrm{gw}}=E_{\mathrm{rot}}$ at each $\left(n, f_{\text {start }}, \tau\right)$ parameter point, assuming a NS moment of inertia of $I_{z z}=100 M_{\odot}^{3} G^{2} / c^{4} \approx 4.34 \times 10^{38} \mathrm{~kg} \mathrm{~m}^{2}$. Injections were randomized over source inclination cos $\iota$ for HMM and FreqHough, while for STAMP and ATrHough injections for the best case $(\cos \iota=1)$ and worst case $(\cos \iota=0)$ are shown separately. For comparison, the known distance to the source of GW170817 is indicated by a horizontal dashed line in the left-hand panel, as well as two (optimistic) energy upper limits in the right-hand panel: the total system energy (dotted line, using a fiducial value of $E_{\text {tot }}=3.265 M_{\odot} c^{2}$ as in Abbott et al. 2017g) and the initial rotational energy $E_{\text {rot }}$ as a function of $f_{\text {start }}$ (dashed line).
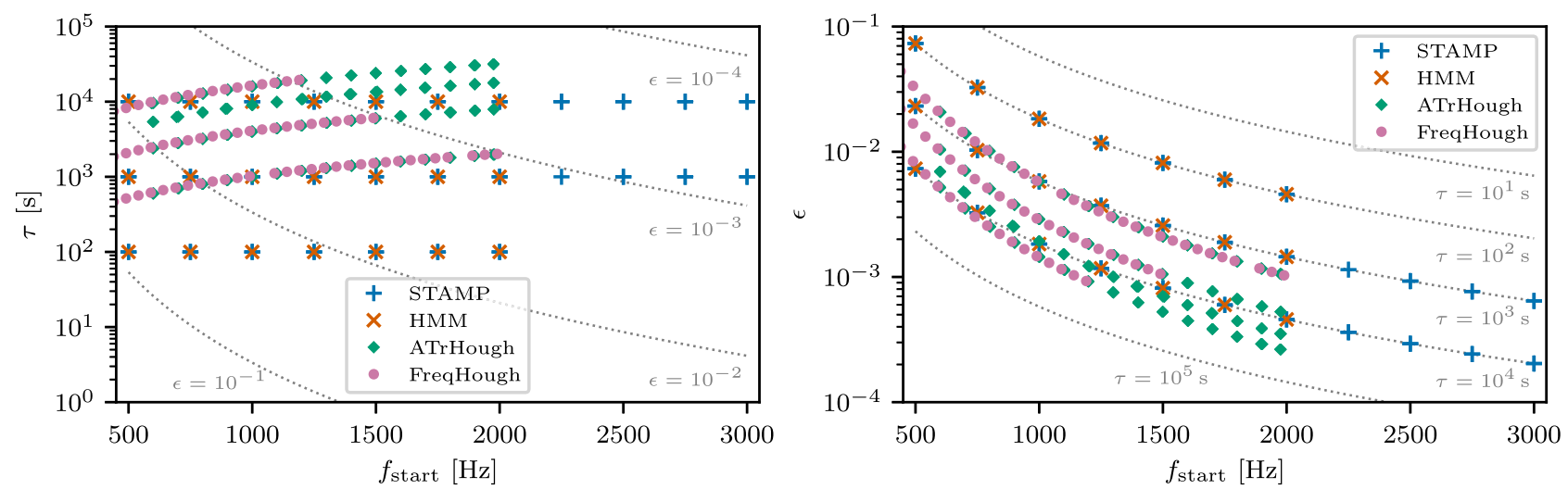

Figure 3. Parameter coverage in $f_{\text {start }}, \tau$ and $\epsilon$ of the injection sets used for the $n=5$ sensitivity estimates, as listed in Tables $2-5$. As shown in the left-hand panel, the HMM and STAMP injections are at fixed $\tau \in\left[10^{2}, 10^{3}, 10^{4}\right] \mathrm{s}$, while for ATrHough and FreqHough different $\tau\left(f_{\text {start }}\right)$ curves are covered for different choices of $T_{\text {SFT }}$ (and, in the case of FreqHough, $\Delta t)$ in the search setup. At each $\left(n, f_{\text {start }}, \tau\right)$ parameter space point, the maximum $\epsilon$ allowed by the energy budget $\left(E_{\mathrm{gw}}=E_{\text {rot }}\right)$ is chosen (right-hand panel), assuming a NS moment of inertia of $I_{z z}=100 M_{\odot}^{3} G^{2} / c^{4} \approx 4.34 \times 10^{38} \mathrm{~kg} \mathrm{~m}^{2}$. Lines of constant $\epsilon$ (left panel) or $\tau$ (right-hand panel) are shown for comparison. STAMP injections include $f_{\text {start }}$ up to $3000 \mathrm{~Hz}$ for longer $\tau$, with those above $2000 \mathrm{~Hz}$ covered by the high-frequency search configuration. But for $\tau=100 \mathrm{~s}$, we limit $f_{\text {start }}$ to $2000 \mathrm{~Hz}$ because injections at higher frequencies would leave the high-frequency band too rapidly to be recoverable.

model parameters $\left\{n=5, f_{\text {start }}, \tau\right\}$, we consider the maximum $\epsilon$ allowed by the initial rotational energy budget (Sarin et al. 2018): the total emitted GW energy as $t \rightarrow \infty$,

$$
E_{\mathrm{gw}}=-\int_{t=t_{\mathrm{start}}}^{\infty} d t \frac{32 G}{5 c^{5}} I_{z z}^{2} \epsilon^{2} \Omega^{6}(t)
$$

must not exceed the remnant's initial rotational energy $E_{\text {rot }}=$ $0.5 I_{z z} f_{\text {start }}^{2} \pi^{2}$.

Given each pipeline's detection threshold, we can rescale the amplitude of simulated signals until $90 \%$ of them are recovered above threshold, while randomizing over nuisance parameters (polarization angle and initial phase of the signal; also source inclination $\iota$ and signal start time for some of the pipelines). We can then either interpret this amplitude scaling as a need to lower the distance of simulated sources; i.e., estimating the sensitive distance $d^{90 \%}$ of the search. Alternatively, we can fix the true distance to the source of GW170817 to obtain an energy upper limit $E_{\mathrm{gw}}^{90 \%}$ : for a remnant NS with the given parameters, we interpret the square of the amplitude scaling as the factor by which the energy output needs to be greater than the expected amount to produce signals that we can recover at $90 \%$ confidence. Both interpretations are shown in Figure 2, with coverage of the injection sets illustrated in Figure 3. The full results are listed in the appendix in Tables 2-5.

The highest sensitivities are achieved at low $f_{\text {start }}$ and for rapid spindown (low $\tau$ ). This is mostly due to the energy budget constraint enforced on $\epsilon$ : in principle, higher $f_{\text {start }}$ yield higher initial amplitudes and longer $\tau$ allow accumulation of $\mathrm{S} / \mathrm{N}$ over longer observation times $\left(\mathrm{S} / \mathrm{N} \propto \tau^{1 / 2}\right.$ for $n=5$ and once $T_{\mathrm{obs}}>\tau$ ). However, due to the energy budget constraint, $\epsilon$ must be lower in this region of parameter space and hence actual detectability is reduced ( $\mathrm{S} / \mathrm{N} \propto \epsilon \propto \tau^{-1} f_{\text {start }}^{-6}$ ).

The four pipelines perform differently across $\tau$ regimes: the unmodeled STAMP and HMM are most sensitive at the shortest $\tau=10^{2} \mathrm{~s}$, but lose up to an order of magnitude in $d^{90 \%}$ when going to $\tau=10^{4} \mathrm{~s}$. Meanwhile, the model-based semi-coherent 
Table 2

STAMP Search Sensitivities Estimated from Simulated Signals (Injections) Following the Power-law Spin-down Model with Braking Index $n=5$

\begin{tabular}{|c|c|c|c|c|c|}
\hline $\cos \iota$ & $f_{\mathrm{gw} 0}(\mathrm{~Hz})$ & $\tau(\mathrm{s})$ & $\epsilon$ & $d^{90 \%}(\mathrm{Mpc})$ & $E_{\mathrm{gw}}^{90 \%}\left(M_{\odot} c^{2}\right)$ \\
\hline 0.0 & 500 & $1.00 \times 10^{2}$ & $7.33 \times 10^{-2}$ & $0.14_{-0.03}^{+0.07}$ & $2.39_{-0.88}^{+2.85} \times 10^{2}$ \\
\hline 0.0 & 750 & $1.00 \times 10^{2}$ & $3.26 \times 10^{-2}$ & $0.055_{-0.017}^{+0.047}$ & $3.52_{-1.80}^{+8.43} \times 10^{3}$ \\
\hline 0.0 & 1000 & $1.00 \times 10^{2}$ & $1.83 \times 10^{-2}$ & $0.12_{-0.02}^{+0.05}$ & $1.25_{-0.42}^{+1.25} \times 10^{3}$ \\
\hline 0.0 & 1250 & $1.00 \times 10^{2}$ & $1.17 \times 10^{-2}$ & $0.12_{-0.02}^{+0.04}$ & $2.10_{-0.58}^{+1.57} \times 10^{3}$ \\
\hline 0.0 & 1500 & $1.00 \times 10^{2}$ & $8.14 \times 10^{-3}$ & $0.090_{-0.017}^{+0.038}$ & $5.36_{-1.81}^{+5.45} \times 10^{3}$ \\
\hline$\cdots$ & $\cdots$ & $\cdots$ & $\cdots$ & $\cdots$ & $\cdots$ \\
\hline
\end{tabular}

Note. Each row corresponds to an injection set with fixed parameters; sensitivities are at $90 \%$ confidence.

(This table is available in its entirety in machine-readable form.)

Table 3

HMM Search Sensitivities Estimated from Simulated Signals (Injections) Following the Power-law Spin-down Model with Braking Index $n=5$

\begin{tabular}{lcccc}
\hline \hline$f_{\mathrm{gw} 0}(\mathrm{~Hz})$ & $\tau(\mathrm{s})$ & $\epsilon$ & $d^{90 \%}(\mathrm{Mpc})$ & $E_{\mathrm{gw}}^{90 \%}\left(M_{\odot} c^{2}\right)$ \\
\hline 500 & $1.00 \times 10^{2}$ & $7.33 \times 10^{-2}$ & $0.86_{-0.16}^{+0.16}$ & $6.51_{-1.87}^{+3.29} \times 10^{0}$ \\
750 & $1.00 \times 10^{2}$ & $3.26 \times 10^{-2}$ & $0.61_{-0.07}^{+0.07}$ & $2.92_{-0.58}^{+0.82} \times 10^{1}$ \\
1000 & $1.00 \times 10^{2}$ & $1.83 \times 10^{-2}$ & $0.48_{-0.04}^{+0.04}$ & $8.43_{-1.25}^{+1.60} \times 10^{1}$ \\
1250 & $1.00 \times 10^{2}$ & $1.17 \times 10^{-2}$ & $0.40_{-0.03}^{+0.03}$ & $1.90_{-0.22}^{+0.27} \times 10^{2}$ \\
1500 & $1.00 \times 10^{2}$ & $8.14 \times 10^{-3}$ & $0.26_{-0.02}^{+0.02}$ & $6.15_{-0.75}^{+0.91} \times 10^{2}$ \\
$\ldots$ & $\ldots$ & $\ldots$ & $\ldots$ & $\ldots$ \\
\hline
\end{tabular}

Note. Each row corresponds to injections marginalized over random $\cos \iota$; sensitivities are at $90 \%$ confidence.

(This table is available in its entirety in machine-readable form.)

ATrHough and FreqHough have focused on longer $\tau$ of $4 \times 10^{2} \mathrm{~s}$ to $3 \times 10^{4} \mathrm{~s}$, with only up to a factor of 2 loss in $d^{90 \%}$ for the longest $\tau$ at fixed $f_{\text {start }}$. See Figures $6-9$ in the appendix for sensitivity estimates over each pipeline's full injection set.

Given that this parameter dependence is shaped by the $E_{\mathrm{gw}}=E_{\mathrm{rot}}$ constraint and is also influenced by some practical tradeoffs in pipeline configuration, in this paper we do not attempt to provide a general evaluation of pipeline performance on fully equivalent injection sets or for generic GW signals. Such a comparison would require a detailed mock data challenge similar to Messenger et al. (2015) and Walsh et al. (2016). Instead, Figure 2 shows results from each pipeline for the parts of parameter space where it achieved its highest sensitivity.

In summary, in no part of the $n=5$ parameter space covered by the four search ranges and injection sets do we reach $90 \%$ sensitive distances of $1 \mathrm{Mpc}$ or further. This corresponds to a lowest $90 \%$ detectable energy of $E_{\mathrm{gw}} \lesssim 8 M_{\odot} c^{2}$ at $f_{\text {start }}=$ $500 \mathrm{~Hz}$ and $\tau=100 \mathrm{~s}$. At higher $f_{\text {start }}$, the sensitive distances for any $\tau$ are even lower due to the energy constraint. Note again that this covers power-law spindown signals, both starting immediately at coalescence time $t_{\mathrm{c}}$ and signals starting with some time delay, with a delay time of 1-7 hr for FreqHough and any possible delay time until the end of $\mathrm{O} 2$ for STAMP.

At the shortest $\tau$, the parameter space covered here overlaps with the magnetar injections in the shorter-duration search of Abbott et al. (2017g), ${ }^{172}$ although the results in that paper were

\footnotetext{
${ }^{172}$ We note here a mistake in Abbott et al. (2017g): in Section 3.2.4, the equivalent energies for the best STAMP results should have read $E_{\mathrm{gw}} \approx 0.6 M_{\odot} c^{2}$ for bar modes and $E_{\mathrm{gw}} \approx 10 M_{\odot} c^{2}$ for the magnetar model, instead of the quoted 2 and $4 M_{\odot} c^{2}$. The corresponding $h_{\text {rss }}$ values in the text of Abbott et al. (2017g) and in its Tables 2 and 3, as well as Figure 1, are correct as published.
}

quoted as recoverable at $50 \%$ confidence, and hence are more optimistic than the new results at $90 \%$. For example, at $f_{\text {start }}=1000 \mathrm{~Hz}$ and $\tau=100 \mathrm{~s}$, the STAMP analysis in the previous paper found $E_{\mathrm{gw}}^{50 \%} \approx 24 M_{\odot} c^{2}$ while the new STAMP and $\mathrm{HMM}$ analyses presented here obtain $E_{\mathrm{gw}}^{90 \%} \approx 100 M_{\odot} c^{2}$ at these parameters. For the pipelines in this paper, amplitudes for detectability at $50 \%$ confidence are typically lower by a factor of 2-4 than those at $90 \%$ confidence. Although these lower thresholds would push the best $d^{50 \%}$ limits up to a few Mpc, this would not change the conclusion that any GWs from a long-lived remnant of GW170817 at $40 \mathrm{Mpc}$ would be undetectable.

\section{Conclusion}

We have searched for the GW emission from a putative remnant neutron star of the BNS merger GW170817, concentrating on signals lasting from hundreds of seconds upwards and described by a power-law spin-down model. However, two of the four employed analysis methods were designed to be sensitive to any generic signal morphology in the covered observation time. In keeping with the available energy budget and theoretical sensitivity estimates, we have not found any significant signal candidates. Studies with simulated signals confirm that we would have only been sensitive to a signal from GW-dominated spin-down (at the time and sky location of GW170817) for distances of less than $1 \mathrm{Mpc}$, or equivalently for unphysical amounts of emitted GW energy.

The four analysis pipelines that have been used in this work have complementary strengths in parameter space coverage, and also in their response to noise artifacts and gaps in the data. While further development of these methods is expected, improvements are also needed — and already in progress-on the instrumentation side. Ongoing instrumental enhancements of aLIGO and Virgo to improve their design sensitivies (Abbott et al. 2018d), and further upgrades such as LIGO $\mathrm{A}+$ (Barsotti et al. 2018) in the next decade, will improve strain sensitivity across the detector band. Improved highfrequency performance is of particular importance for postmerger searches because the highest signal amplitudes are emitted in the early, high-frequency part of the spin-down, where the detectors are currently much less sensitive than around a few hundred Hz. The search for long-duration postmerger signals from supramassive or stable NSs could then enter into the astrophysically constraining regime. However, even if we scale the sensitivies obtained in this analysis (or even those estimated for an optimal matched-filter analysis by Sarin et al. 2018) with the expected improvements of 2-4 in strain, they will still be limited to the most nearby BNS events. 
Table 4

ATrHough Search Sensitivities Estimated from Simulated Signals (Injections) Following the Power-law Spin-down Model with Braking Index $n=5$

\begin{tabular}{|c|c|c|c|c|c|c|}
\hline$T_{\mathrm{SFT}}(\mathrm{s})$ & $\cos \iota$ & $f_{\mathrm{gw} 0}(\mathrm{~Hz})$ & $\tau(\mathrm{s})$ & $\epsilon$ & $d^{90 \%}(\mathrm{Mpc})$ & $E_{\mathrm{gw}}^{90 \%}\left(M_{\odot} c^{2}\right)$ \\
\hline 2 & 0.0 & 601 & $6.01 \times 10^{2}$ & $2.07 \times 10^{-2}$ & $0.086_{-0.012}^{+0.009}$ & $9.30_{-2.32}^{+1.96} \times 10^{2}$ \\
\hline 2 & 0.0 & 700 & $7.00 \times 10^{2}$ & $1.41 \times 10^{-2}$ & $0.053_{-0.012}^{+0.010}$ & $3.32_{-1.37}^{+1.29} \times 10^{3}$ \\
\hline 2 & 0.0 & 800 & $8.00 \times 10^{2}$ & $1.01 \times 10^{-2}$ & $0.061_{-0.010}^{+0.008}$ & $3.32_{-1.03}^{+0.89} \times 10^{3}$ \\
\hline 2 & 0.0 & 898 & $8.98 \times 10^{2}$ & $7.57 \times 10^{-3}$ & $0.065_{-0.008}^{+0.006}$ & $3.66_{-0.81}^{+0.65} \times 10^{3}$ \\
\hline 2 & 0.0 & 1000 & $1.00 \times 10^{3}$ & $5.80 \times 10^{-3}$ & $0.069_{-0.008}^{+0.006}$ & $4.07_{-0.89}^{+0.73} \times 10^{3}$ \\
\hline$\cdots$ & $\cdots$ & $\cdots$ & $\cdots$ & $\cdots$ & $\ldots$ & $\cdots$ \\
\hline
\end{tabular}

Note. Each row corresponds to injections marginalized over a small band in $f_{\mathrm{gw} 0}$; Sensitivities are at $90 \%$ confidence.

(This table is available in its entirety in machine-readable form.)

Table 5

FreqHough Search Sensitivities Estimated from Simulated Signals (Injections) Following the Power-law Spin-down Model with Braking Index $n=5$

\begin{tabular}{|c|c|c|c|c|c|}
\hline$T_{\mathrm{SFT}}(\mathrm{s})$ & $f_{\text {start }}(\mathrm{Hz})$ & $\tau(\mathrm{s})$ & $\epsilon$ & $d^{90 \%}(\mathrm{Mpc})$ & $E_{\mathrm{gw}}^{90 \%}\left(M_{\odot} c^{2}\right)$ \\
\hline 2 & 390 & $4.15 \times 10^{2}$ & $5.91 \times 10^{-2}$ & $0.78_{-0.04}^{+0.05}$ & $4.76_{-0.50}^{+0.59} \times 10^{0}$ \\
\hline 2 & 440 & $4.65 \times 10^{2}$ & $4.39 \times 10^{-2}$ & $0.83_{-0.05}^{+0.06}$ & $5.42_{-0.62}^{+0.75} \times 10^{0}$ \\
\hline 2 & 490 & $5.15 \times 10^{2}$ & $3.36 \times 10^{-2}$ & $0.79_{-0.05}^{+0.06}$ & $7.39_{-0.89}^{+1.08} \times 10^{0}$ \\
\hline 2 & 540 & $5.65 \times 10^{2}$ & $2.64 \times 10^{-2}$ & $0.72_{-0.04}^{+0.05}$ & $1.06_{-0.12}^{+0.15} \times 10^{1}$ \\
\hline 2 & 590 & $6.15 \times 10^{2}$ & $2.12 \times 10^{-2}$ & $0.75_{-0.05}^{+0.06}$ & $1.19_{-0.16}^{+0.20} \times 10^{1}$ \\
\hline$\cdots$ & $\cdots$ & $\cdots$ & $\cdots$ & $\ldots$ & $\ldots$ \\
\hline
\end{tabular}

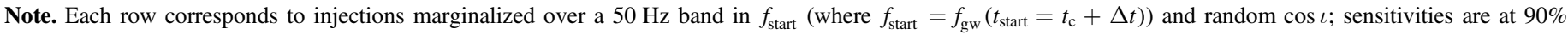
confidence.

(This table is available in its entirety in machine-readable form.)

Third generation detectors, such as the Einstein Telescope (Hild et al. 2011; Sathyaprakash et al. 2012) and Cosmic Explorer (Abbott et al. 2017b), promise a strain sensitivity increase of $\sim 20-30$ over aLIGO at design sensitivity. Consequently, GWs from a long-lived remnant of another BNS at the same distance as GW170817 should then become observable.

The authors gratefully acknowledge the support of the United States National Science Foundation (NSF) for the construction and operation of the LIGO Laboratory and Advanced LIGO and also the Science and Technology Facilities Council (STFC) of the United Kingdom, the Max-Planck-Society (MPS), and the State of Niedersachsen/Germany for support of the construction of Advanced LIGO and construction and operation of the GEO600 detector. Additional support for Advanced LIGO was provided by the Australian Research Council. The authors gratefully acknowledge the Italian Istituto Nazionale di Fisica Nucleare (INFN), the French Centre National de la Recherche Scientifique (CNRS) and the Foundation for Fundamental Research on Matter supported by the Netherlands Organisation for Scientific Research, for the construction and operation of the Virgo detector and the creation and support of the EGO consortium. The authors also gratefully acknowledge research support from these agencies as well as by the Council of Scientific and Industrial Research of India, the Department of Science and Technology, India, the Science \& Engineering Research Board (SERB), India, the Ministry of Human Resource Development, India, the Spanish Agencia Estatal de Investigación, the Vicepresidència i Conselleria d'Innovació Recerca i Turisme and the Conselleria d'Educació i Universitat del Govern de les Illes Balears, the Conselleria d'Educació Investigació Cultura i Esport de la Generalitat Valenciana, the National Science Centre of Poland, the Swiss National Science Foundation (SNSF), the Russian Foundation for Basic Research, the Russian Science Foundation, the European Commission, the European Regional Development Funds (ERDF), the Royal Society, the Scottish Funding Council, the Scottish Universities Physics Alliance, the Hungarian Scientific Research Fund (OTKA), the Lyon Institute of Origins (LIO), the Paris Île-de-France Region, the National Research, Development and Innovation Office Hungary (NKFI), the National Research Foundation of Korea, Industry Canada and the Province of Ontario through the Ministry of Economic Development and Innovation, the Natural Science and Engineering Research Council Canada, the Canadian Institute for Advanced Research, the Brazilian Ministry of Science, Technology, Innovations, and Communications, the International Center for Theoretical Physics South American Institute for Fundamental Research (ICTP-SAIFR), the Research Grants Council of Hong Kong, the National Natural Science Foundation of China (NSFC), the Leverhulme Trust, the Research Corporation, the Ministry of Science and Technology (MOST), Taiwan and the Kavli Foundation. The authors gratefully acknowledge the support of the NSF, STFC, MPS, INFN, CNRS and the State of Niedersachsen/Germany for provision of computational resources. This article has been assigned document number LIGO-P1800195.

\section{Appendix A Additional Details on Search Methods}

\section{A.1. STAMP}

Spectrogram pixel sizes. The low-frequency band from 30 to $2000 \mathrm{~Hz}$ uses pixels of $100 \mathrm{~s} \times 1 \mathrm{~Hz}$, while the high-frequency band from 2000 to $4000 \mathrm{~Hz}$ uses pixels of smaller durations of 


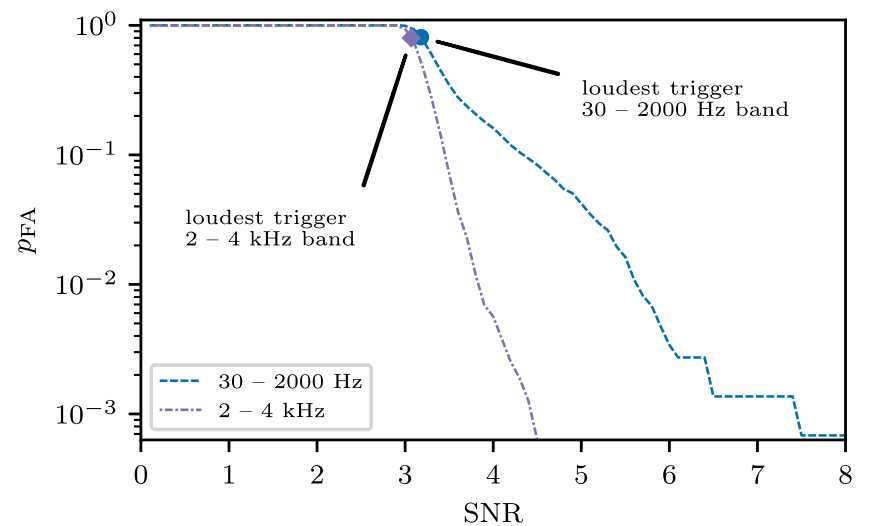

Figure 4. STAMP background distributions, in terms of false-alarm probability $p_{\mathrm{FA}}$ as a function of detection statistic $(\mathrm{S} / \mathrm{N})$, for the low- and high-frequency bands, and the corresponding loudest foreground triggers (dot and diamond symbols).

$50 \mathrm{~s} \times 1 \mathrm{~Hz}$. Smaller pixels at higher frequency are necessary to account for the rotation of the Earth, which causes the GW phase difference between detectors to change with time. There is a loss of $\mathrm{S} / \mathrm{N}$ when the pixel durations are too large, which increases with frequency. Therefore, the durations are chosen to limit the maximum possible $\mathrm{S} / \mathrm{N}$ loss in a pixel (at the highest frequencies) from this effect to about 10\% (Thrane et al. 2015).

Detection statistic. Each spectrogram in the STAMP search (Thrane et al. 2011; Thrane \& Coughlin 2013; Thrane et al. 2015) is analyzed with many randomly chosen quadratic Bézier curves. The $\mathrm{S} / \mathrm{N}$ of each track $\rho_{\Gamma}$ is a weighted sum of the $\mathrm{S} / \mathrm{N}$ of the pixels covered by the track. The quantity $\rho_{\Gamma}$ also serves as the detection statistic and is calculated as:

$$
\rho_{\Gamma}=\frac{1}{N^{3 / 4}} \sum_{i} \rho_{i},
$$

where $i$ runs over all the pixels in a track and $N$ is the total number of pixels in it. These are then ranked and the track with largest $\rho_{\Gamma}$ is picked as the trigger for a map. This is done for both the main on-source search and for the background estimation over time-shifted data.

Background triggers and loudest events. Figure 4 shows the distribution of false-alarm probabilities $p_{\mathrm{FA}}$ for the $\mathrm{S} / \mathrm{Ns}$ of triggers collected in background data, for both high- and lowfrequency spectrograms. The loudest on-source event in each frequency range is also shown.

\section{A.2. HMM Tracking}

A general description of the HMM method is given by Suvorova et al. (2016) and Sun et al. (2018). The following summary is intended to clarify the configuration used for the search presented in this paper.

Probabilistic model. A Markov chain is a stochastic process transitioning between discrete states at discrete times $\left\{t_{0}, \cdots, t_{N_{T}}\right\}$. A HMM is an automaton based on a Markov chain, composed of the hidden (unmeasurable) state variable $q(t) \in\left\{q_{1}, \cdots, q_{N_{Q}}\right\}$ and the measurement variable $o(t) \in\left\{o_{1}, \cdots, o_{N_{O}}\right\}$. A HMM is memoryless; i.e., the hidden state at time $t_{n+1}$ only depends on the state at time $t_{n}$, with transition probability

$$
A_{q_{j} q_{i}}=P\left[q\left(t_{n+1}\right)=q_{j} \mid q\left(t_{n}\right)=q_{i}\right] .
$$

The hidden state $q_{i}$ is in observed state $o_{j}$ at time $t_{n}$ with emission probability

$$
L_{o_{j} q_{i}}=P\left[o\left(t_{n}\right)=o_{j} \mid q\left(t_{n}\right)=q_{i}\right] .
$$

Given the prior defined by

$$
\Pi_{q_{i}}=P\left[q\left(t_{0}\right)=q_{i}\right],
$$

the probability that the hidden state path $Q=\left\{q\left(t_{0}\right), \cdots, q\left(t_{N_{T}}\right)\right\}$ gives rise to the observed sequence $O=\left\{o\left(t_{0}\right), \cdots, o\left(t_{N_{T}}\right)\right\}$ equals

$$
P(Q \mid O)=L_{o\left(t_{N_{T}}\right) q\left(t_{N_{T}}\right)} A_{q\left(t_{N_{T}}\right) q\left(t_{N_{T}-1}\right)} \cdots L_{o\left(t_{1}\right) q\left(t_{1}\right)} A_{q\left(t_{1}\right) q\left(t_{0}\right)} \Pi_{q\left(t_{0}\right)} .
$$

The most probable path

$$
Q^{*}(O)=\operatorname{argmax} P(Q \mid O),
$$

maximizes $P(Q \mid O)$ and gives the best estimate of $q(t)$ over the total observation, where $\operatorname{argmax}(\cdots)$ returns the argument that maximizes the function $(\cdots)$. We use the classic Viterbi algorithm (Viterbi 1967) to efficiently solve the HMM and compute $Q^{*}(O)$.

Search setup. In this analysis, we track $q(t)=f_{\mathrm{gw}}(t)$, where $f_{\mathrm{gw}}(t)$ is the GW signal frequency at time $t$. The discrete hidden states are mapped one-to-one to the frequency bins in the output of a frequency-domain estimator $G(f)$ computed over an interval of length $T_{\text {drift }}$, with bin size $\Delta f$. We aim to search for signals with $10^{2} \mathrm{~s} \lesssim \tau \lesssim 10^{4} \mathrm{~s}$, corresponding to $\dot{f}_{\mathrm{gw}} \lesssim 1 \mathrm{~Hz} \mathrm{~s}^{-1}$. Therefore, we choose $T_{\text {drift }}=1 \mathrm{~s}$ (i.e., $\Delta f=1 \mathrm{~Hz}$ ) to satisfy

$$
\left|\int_{t}^{t+T_{\mathrm{drift}}} d t^{\prime} \dot{f}_{\mathrm{gw}}\left(t^{\prime}\right)\right| \leqslant \Delta f
$$

for $0 \leqslant t \leqslant T_{\mathrm{obs}}$, where $T_{\mathrm{obs}}$ is the total observing time. The motion of the Earth with respect to the SSB can be neglected during the interval $\left[t, t+T_{\text {drift }}\right]$. Hence, the emission probability $L_{o(t) q_{i}}=P\left[o(t) \mid f_{i} \leqslant f_{\mathrm{gW}}(t) \leqslant f_{i}+\Delta f\right] \propto \exp \left[G\left(f_{i}\right)\right]$ is calculated from the running-mean (window width $3 \mathrm{~Hz}$ ) normalized power in SFTs with length $T_{\mathrm{SFT}}=T_{\text {drift }}=1 \mathrm{~s}$ as the estimator $G(f)$. We write

$$
G\left(f_{i}\right)=\sum_{X} \tilde{y}_{i}^{X} \tilde{y}_{i}^{X *},
$$

where $i$ indexes the frequency bins of the normalized SFT $\tilde{y}, X$ indexes the detector, and the repeated index $i$ on the right-hand side does not imply summation. We assume that the autocorrelation timescale of timing noise is much longer than $T_{\text {drift }}$, and hence adopt the transition probabilities

$$
A_{q_{i-1} q_{i}}=A_{q_{i} q_{i}}=\frac{1}{2},
$$

with all other entries being zero. Since we have no independent knowledge of $f_{\mathrm{gw}}$, we choose a uniform prior, viz

$$
\Pi_{q_{i}}=N_{Q}^{-1} \text {. }
$$

We define a detection statistic $\mathcal{P}$, given by

$$
\mathcal{P}=\frac{1}{N_{T}+1} \sum_{n=0}^{N_{T}} G\left[f_{i\left(t_{n}\right)}\right],
$$

where the integer $i\left(t_{n}\right)$ indexes the SFT frequency bin corresponding to $q^{*}\left(t_{n}\right)$ on the optimal path $Q^{*}\left(t_{0} \leqslant t_{n} \leqslant t_{N_{T}}\right)$. 
The strain amplitude $h_{0}$ in (4) decreases significantly for $t \gg \tau$. Hence, the instant $\mathrm{S} / \mathrm{N}$ decreases for $T_{\mathrm{obs}} \gtrsim \tau$. MonteCarlo simulations show that choosing $T_{\text {obs }} \sim \tau$ yields the best sensitivities for signals with $h_{0}$ (Equation (4)) near the detection limit for the waveform in Equation (3).

The initial spin-down rate $\left|\dot{f}_{\mathrm{gw} 0}\right|$ of a signal with $\tau \lesssim 10^{3} \mathrm{~s}$ can be too high (i.e., $\left|\dot{f}_{\mathrm{gw} 0}\right|>1 \mathrm{~Hz} \mathrm{~s}^{-1}$ ) for Equation (13) to be satisfied with $T_{\text {drift }}=1 \mathrm{~s}$. We start the search after waiting for a time $t_{\text {wait }}$ post-merger, when $\dot{f}_{\mathrm{gw}} \mid$ decreases such that Equation (13) is satisfied. Alternatively, we can choose shorter $T_{\text {drift }}$ (i.e., $T_{\text {drift }} \leqslant \dot{f}_{\mathrm{gw}}^{-1 / 2}$ ) and take $t_{\text {wait }}=0$ for all waveforms. However, the sensitivity degrades because the frequency resolution $\Delta f>1 \mathrm{~Hz}$ is relatively coarse for $T_{\mathrm{drift}}<1 \mathrm{~s}$.

In a search without prior knowledge of the signal model, we cover the following parameter space $500 \mathrm{~Hz} \leqslant f_{\mathrm{gw} 0} \leqslant 2 \mathrm{kHz}$ for $10^{2} \mathrm{~s} \lesssim \tau \lesssim 10^{4} \mathrm{~s}$ using seven discrete $t_{\text {wait }}$ values in the range $0 \leqslant t_{\text {wait }} \leqslant 400 \mathrm{~s}$. Monte-Carlo simulations show that the impact on sensitivity from the mismatch in $t_{\text {wait }}$ caused by the granularity is negligible.

\section{A.3. Adaptive Transient Hough}

We summarize here some of the key technical details concerning the search at hand, while the complete derivation of the search method is in Oliver et al. (2019).

Coherence times. The first step is to select the coherent integration time $T_{\mathrm{SFT}}$; i.e., the time-baseline of the SFTs. This cannot be arbitrarily large: to avoid the signal shifting more than half a frequency bin, $T_{\mathrm{SFT}}$ must satisfy $\left|\dot{f}_{\mathrm{gw}}\right| T_{\mathrm{SFT}} \leqslant$ $1 /\left(2 T_{\mathrm{SFT}}\right)$. The time variation of $f_{\mathrm{gw}}(t)$ is due to two effects: the spin-down of the source, and the Doppler modulation due to the Earth's motion. It is important to notice that in contrast to the continuous wave case, this method assumes that the Doppler modulation is a subdominant effect. Thus, $T_{\mathrm{SFT}}$ can be estimated as:

$$
T_{\mathrm{SFT}} \leqslant \frac{\sqrt{(n-1) \tau}}{\sqrt{2 f_{\mathrm{gw} 0}}} .
$$

Hough transform. Second, each of these SFTs is digitized by setting a threshold $\rho_{\text {th }}$ on the normalized power, which is directly related to the false-alarm rate $\alpha$ and false dismissal rate $\beta$ of the search; the optimal value is 1.6 as derived in Krishnan et al. (2004). This digitized spectrum is then weighted based on the noise floor of the detector and the amplitude modulation of the source. The derivation of the weights is given in Oliver et al. (2019) and in Sintes \& Krishnan (2007) for the CW allsky case.

Detection statistic and significance threshold. Finally, each template-defined by the set of signal parameters $\left(f_{\mathrm{gw} 0}, n, \tau\right)$-is incoherently integrated through the appropriate summation, known as the number count, over the weighted digitized spectrum following Equation (3). The critical ratio $\Psi$ is defined to evaluate the significance of a given template, based on the results obtained for the weighted number count and its estimates over Gaussian noise for the mean $\mu$ and the standard deviation $\sigma$ :

$$
\Psi=\frac{n-\mu}{\sigma}=\frac{\sum_{i=1}^{N_{\mathrm{SFTs}}} w_{i} y_{i}-\sum_{i=1}^{N_{\mathrm{SFTs}}} w_{i} \alpha}{\sqrt{\sum_{i=1}^{N_{\mathrm{SFTs}}} w_{i}^{2} \alpha(1-\alpha)}} .
$$

Here, $y_{i}$ corresponds to the $i$ th digitalized bin in a given templated track, $N_{\mathrm{SFTs}}$ is the number of SFTs and the weights are $w_{i} \propto\left(f_{\mathrm{gw}, i}\right)^{2 m}\left(a_{i}^{2}+b_{i}^{2}\right) / S_{\mathrm{n}, i}$, where $a_{i}$ and $b_{i}$ are amplitude functions of the antenna pattern found in (Jaranowski et al. 1998 ) at the $i$ th time step and $S_{\mathrm{n}, i}$ is the power spectral density at that given bin. As mentioned in Sintes \& Krishnan (2007) and Oliver et al. (2019), any change in the normalization of the weights $w_{i}$ will not change the resulting sensitivity and will also leave significances, or critical ratios $\Psi$, unchanged. As shown in Oliver et al. (2019), the critical ratio for a multidetector search can be written as

$$
\Psi_{\mathrm{m}}=\sum_{k=1}^{N_{\mathrm{det}}} \Psi_{k} r_{k}
$$

where $r_{k}$ is each detector's relative contribution ratioproportional to the number of SFTs, the noise power spectral density, the antenna pattern and the signal amplitude patternand $N_{\text {det }}$ is the number of detectors. For detection purposes, a threshold is placed on the two-detector $\Psi_{\mathrm{m}}$ corresponding to a $5 \sigma$ false-alarm probability for the entire template bank. An additional single-detector threshold, used for a consistency veto step, is extrapolated from the $5 \sigma$ threshold on $\Psi_{\mathrm{m}}$, to make the veto safer under consideration of the actual differences in $r_{k}$ for this dataset.

Candidate follow-up. To verify that the 51 outliers found in the initial search step were produced by noise in the detector, and exclude the possibility of having any actual astrophysical signals among them, we performed an additional follow-up step. For each template corresponding to one of the outliers, an analogous analysis was performed but with the template $f_{\mathrm{gw}}(t)$ evolution starting instead $1 \mathrm{hr}$ after the merger. From Equation (3), templates starting at merger time have vanishing overlap with these $+1 \mathrm{hr}$ delayed versions of themselves. For all outliers, we find that the $+1 \mathrm{hr}$ critical ratios are compatible with the results found in the original search within $8 \%$. Given the $5 \sigma$ false-alarm threshold imposed, the critical ratio for these templates can be considered as stationary non-Gaussian noise with a false dismissal probability of less that $10^{-4}$.

\section{A.4. Generalized FrequencyHough}

Full details of the adaptation of the FrequencyHough algorithm (Astone et al. 2014) to the case of rapid-spindown post-merger signals are given in Miller et al. (2018). We summarize here some technical details relevant to the search presented in this paper.

Time offsets and search durations. The search is run in three configurations using varying $T_{\mathrm{SFT}}=2,4,8 \mathrm{~s}$, covering different observing times: $\Delta t=1 \mathrm{hr}$ after merger with $T_{\mathrm{SFT}}=2 \mathrm{~s}$ for signals lasting 700-7000 s, $\Delta t \sim 1.5-3 \mathrm{hr}$ after merger with $T_{\mathrm{SFT}}=4 \mathrm{~s}$ for signals lasting $2000-16000 \mathrm{~s}$, and $\Delta t \sim 2-7 \mathrm{hr}$ after merger with $T_{\mathrm{SFT}}=8 \mathrm{~s}$ for signals lasting $8000-40000 \mathrm{~s}$. The corresponding end times are set separately for each detector to guarantee that the same effective amount of data is covered even in the presence of gaps; the latest timestamps analyzed for either detector are approximately 3,8 and $22 \mathrm{hr}$ after merger in the three configurations (see Figure 5).

Coordinate transformation. The parameters of the powerlaw spindown model are transformed to new coordinates, such that in the new space, the behavior of the signal is linear. If we 


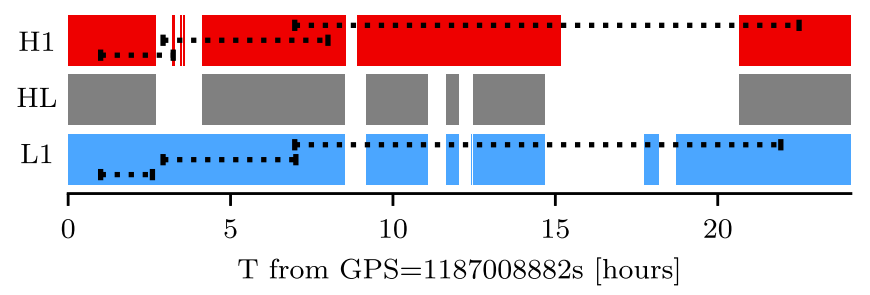

Figure 5. FreqHough: three configurations with different $T_{\mathrm{SFT}}=2,4,8$ were used to search for a signal starting at least one hour to about $20 \mathrm{hr}$ after the merger. The configurations were constructed to maximize sensitivity in different portions of the parameter space. We analyze the same amount of usable data in each detector, regardless of gaps in either detector's data, leading to later end times for the $\mathrm{H} 1$ detector in these three cases.

write $k^{\prime}=(2 \pi)^{n-1} k$, we can rewrite Equation (3) as:

$$
f_{\mathrm{gW}}(t)=\frac{f_{\text {start }}}{\left[1+k^{\prime}(n-1) f_{\text {start }}^{n-1}\left(t-t_{\mathrm{ref}}\right)\right]^{\frac{1}{n-1}}}
$$

where

$$
k^{\prime}=\frac{1}{\tau f_{\text {start }}^{n-1}(n-1)} .
$$

Then we can make the following change of coordinates:

$$
x=\frac{1}{f_{\mathrm{gw}}^{n-1}} ; \quad x_{0}=\frac{1}{f_{\mathrm{start}}^{n-1}} .
$$

Equation (21) becomes the equation of a line:

$$
x=x_{0}+(n-1) k^{\prime}\left(t-t_{0}\right) .
$$

Now, we map peaks in the $\left(t-t_{0}, f_{\mathrm{gw}}\right)$ plane of the detector to lines in the $\left(x_{0}, k\right)$ plane of the source, where both variables in this space relate to the physical parameters of the source.

Grid setup. Our method can be used to determine if a signal is present in the data and estimate its $f_{\text {start }}, \dot{f}_{\text {start }}$ and $n$. We search across different braking indices with a fine, nonuniform grid determined by ensuring that by stepping from $n$ to $n+d n$, a signal is confined to one frequency bin. For each braking index, we do a Hough transform and record the most significant candidates in the map. The grid in $x_{0}$ is determined by taking the derivative of Equation (23); the grid in $k$ is created by considering a transformation $f_{\mathrm{gw}} \rightarrow f_{\mathrm{gw}}+d f_{\mathrm{gw}}=1 / T_{\mathrm{SFT}}$ and $k \rightarrow k+d k$, then solving for $d k$ imposing that the spindown remains constant.

Both grids are nonuniform and depend on the frequency band and spindown range that we use; however, we overresolve the grid in $x_{0}$ for most frequency bands using the maximum frequency we are analyzing because it is computationally faster and does not result in a sensitivity loss.

The transformation has the disadvantage that it creates nonuniform noise (so peaks at higher frequencies contribute more to the Hough map). We account for this by extending the frequency band that we wish to analyze, and we then only select candidates from the original band.

Coincidence step. Candidates are considered in coincidence between detectors if the Euclidean distance between their recovered parameters $x_{0}$ and $k$ is less than 3 bins.

Candidate follow-up. Our candidate follow-up procedure is as follows: we correct for the phase evolution of the candidate recovered in each detector individually. Ideally, if we correct for exactly the right parameters, then we expect a monochromatic signal in the time/frequency peakmap. If we are a bin or so off, then there is a residual spindown or spinup, but the signal is linear. Therefore, we can apply the original FrequencyHough to search for this signal. After applying the original FrequencyHough to the one surviving candidate from this search and performing coincidences again, we found no coincidence, which indicates that the candidate was false. We use the critical ratio as a way to veto candidates, defined as:

$$
\mathrm{CR}=\frac{y-\mu}{\sigma}
$$

where $y$ is the number count in a given bin in the Hough map, $\mu$ is the average number count of the noise, and $\sigma$ is the standard deviation of the number counts due to noise. We determine if the critical ratio increases in the follow-up, but find that it does not for our one remaining candidate.

Sensitivity estimation procedure. We then computed the strain sensitivity for the different configurations of our search in the following way: the loudest coincident candidate was selected in each $50 \mathrm{~Hz}$ band, for $n=5$. Its $f_{\text {start }}$ and duration were used to inject signals with initial frequencies ranging from $f_{\text {start }}$ to $f_{\text {start }}+50 \mathrm{~Hz}$, with the highest possible spindown in our configuration $\dot{f}_{\text {start }}=1 / T_{\mathrm{SFT}}^{2}$. Based on our theoretical estimates for sensitivity, the highest initial spindown corresponds to the most conservative result; i.e., the worst case sensitivity. A total of 100 injections were done for each set of parameters; i.e., each point plotted in Figures 2, 3 and 9 and each row in Table 5. Recovery of an injection is defined in the same way as a detection in the real search: within a coincidence distance of 3 bins.

\section{Appendix B Details on Pipeline Sensitivity}

In Figures 6-9 and Tables 2-5, we summarize the full sensitivity estimates performed for each of the four pipelines 

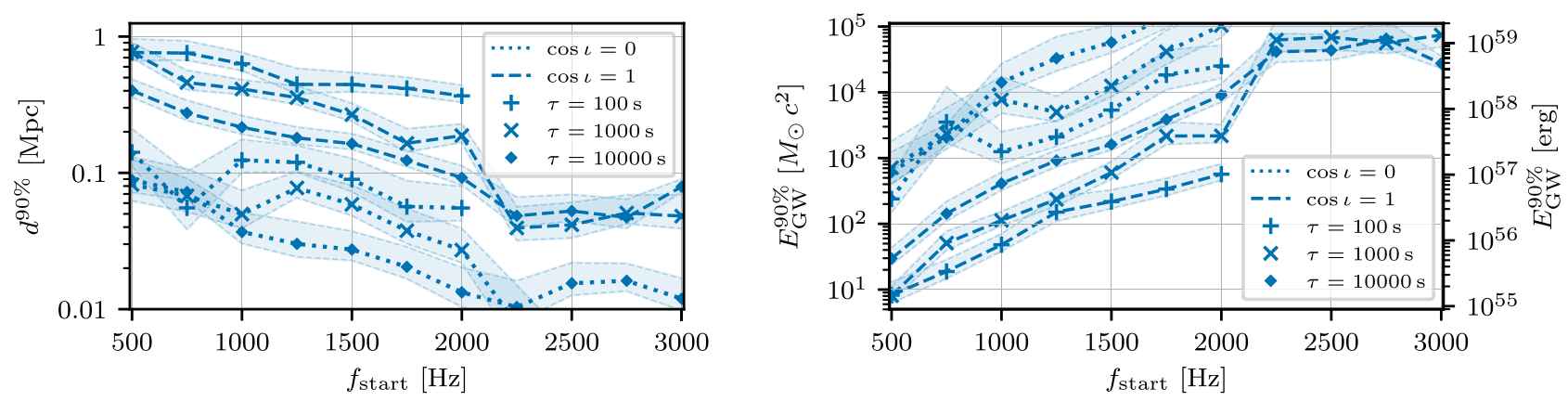

Figure 6. STAMP $90 \%$ sensitivity estimates for $n=5$ and variable $f_{\text {start }}$. For either $\cos \iota=[0,1]$, the connected lines (from top to bottom in $d$ ) are for injections with $\tau \in\left[10^{2}, 10^{3}, 10^{4}\right] \mathrm{s}$. The shaded ranges correspond to $1 \sigma$ binomial counting errors on the injection sets.
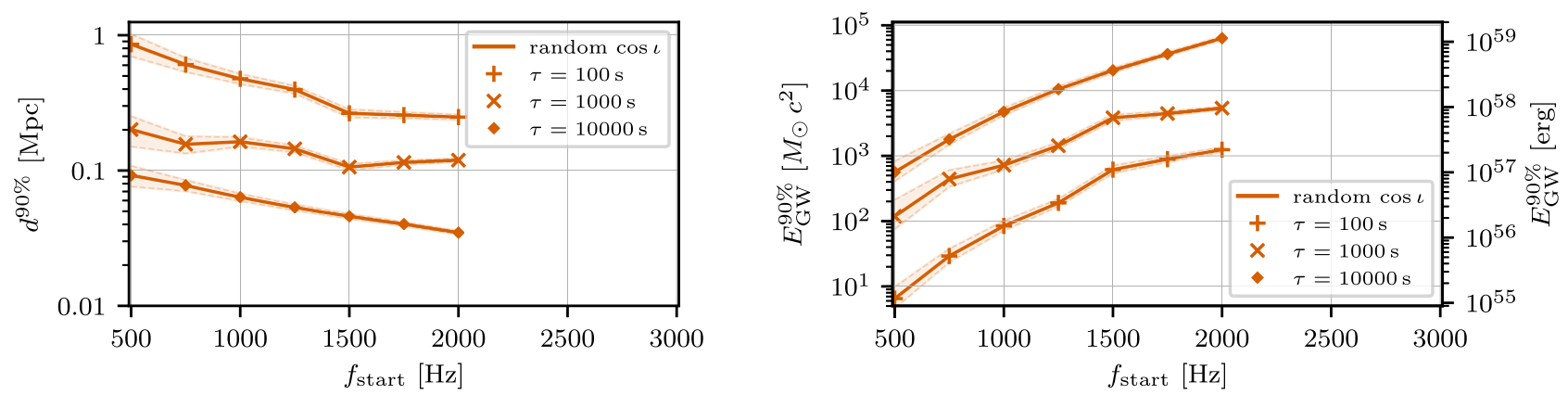

Figure 7. HMM $90 \%$ sensitivity estimates for $n=5$ and $f_{\text {start }}=f_{\mathrm{gw} 0}=f_{\mathrm{gw}}\left(t_{\mathrm{c}}\right.$ ). The connected lines (from top to bottom in $d$ ) are for injections with $\tau \in\left[10^{2}, 10^{3}, 10^{4}\right] \mathrm{s}$. The shaded ranges illustrate the uncertainty due to interpolating discrete steps in injection amplitudes.
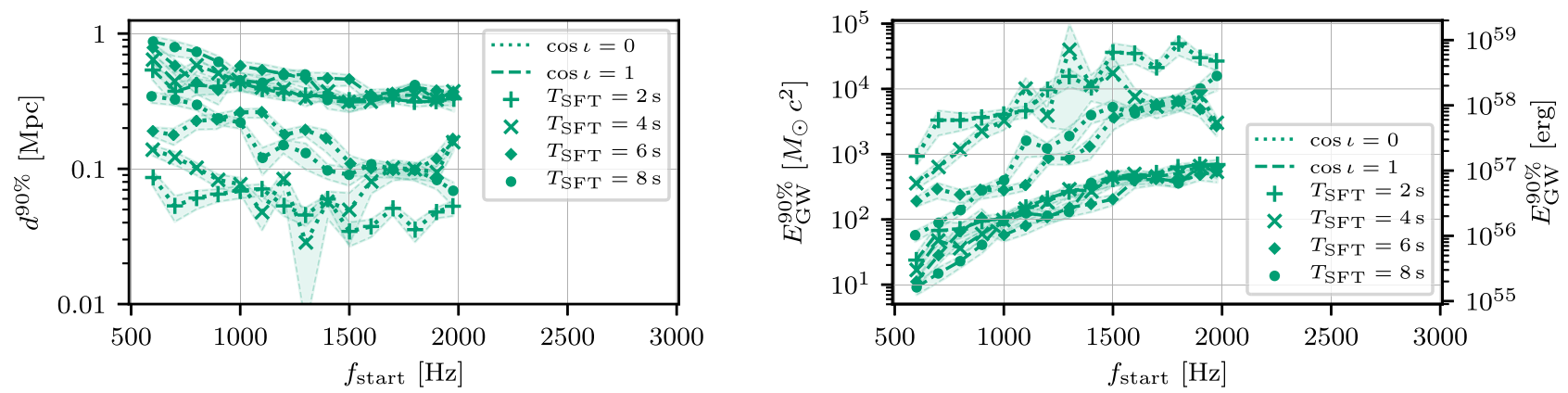

Figure 8. ATrHough $90 \%$ sensitivity estimates for $n=5$ and $f_{\mathrm{start}}=f_{\mathrm{gw} 0}=f_{\mathrm{gw}}\left(t_{\mathrm{c}}\right.$ ). For either $\cos \iota=[0,1]$, the connected lines (from bottom to top in $d$ ) are for coherence times of $T_{\mathrm{SFT}}=[2,4,6,8]$. The shaded ranges: $2 \sigma$ envelopes of logit fits over the different injection sets.
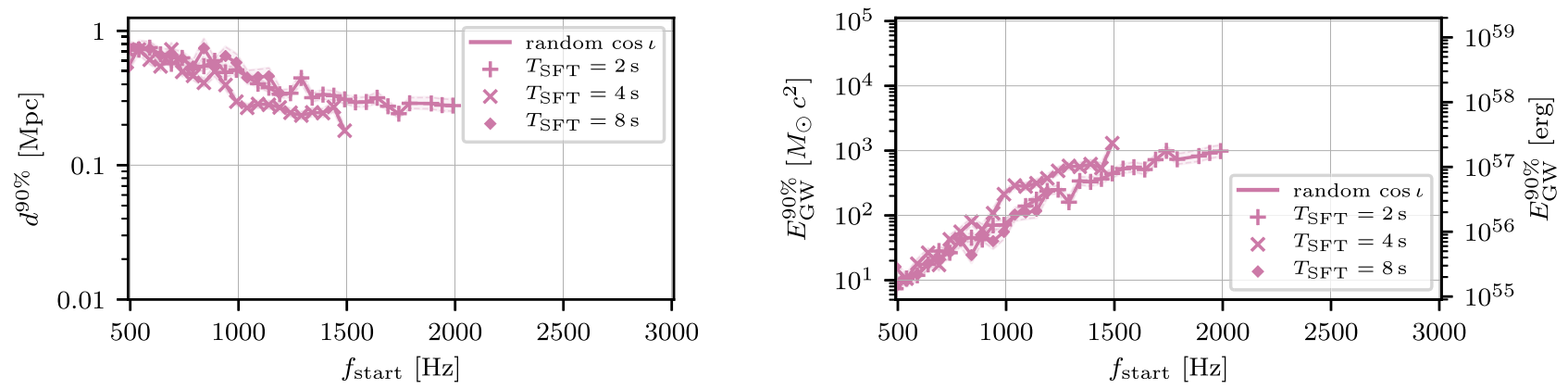

Figure 9. FreqHough $90 \%$ sensitivity estimates for $n=5$ and $f_{\mathrm{start}}=f_{\mathrm{gw}}\left(t_{\mathrm{start}}=t_{\mathrm{c}}+\Delta t\right)$. The connected lines are for coherence times of $T_{\mathrm{SFT}}=[2,4,8] \mathrm{s}$. Shaded ranges give the uncertainty due to interpolating discrete steps in injection amplitudes.

with their respective injection sets of simulated signals following the power-law spin-down model (Equation (3)) with $n=5$. Additional details on the injection sets and sensitivity estimation procedure for each pipeline are given in the previous section or, where necessary, in the table captions. 


\section{ORCID iDs}

K. Ackley (iD https://orcid.org/0000-0002-8648-0767

B. Allen (D) https://orcid.org/0000-0003-4285-6256

F. Aubin (iD https://orcid.org/0000-0002-8241-4156

C. Aulbert (i) https://orcid.org/0000-0002-1481-8319

I. Bartos (iD https://orcid.org/0000-0001-5607-3637

B. Bécsy (iD https://orcid.org/0000-0003-0909-5563

C. Beer (iD https://orcid.org/0000-0003-3991-067X

M. Bejger (i) https://orcid.org/0000-0002-4991-8213

P. Charlton (1D https://orcid.org/0000-0003-0710-1712

R. Ciolfi (i) https://orcid.org/0000-0003-3140-8933

N. Cornish (iD https://orcid.org/0000-0002-7435-0869

A. Corsi (i) https://orcid.org/0000-0001-8104-3536

M. W. Coughlin (10 https://orcid.org/0000-0002-8262-2924

T. Dal Canton (i) https://orcid.org/0000-0001-5078-9044

T. Dent (i) https://orcid.org/0000-0003-1354-7809

Z. Doctor (iD https://orcid.org/0000-0002-2077-4914

H.-B. Eggenstein (1) https://orcid.org/0000-0001-5296-7035

S. Fairhurst (iD https://orcid.org/0000-0001-8480-1961

B. Farr (1) https://orcid.org/0000-0002-2916-9200

W. M. Farr (iD https:// orcid.org/0000-0003-1540-8562

M. Fishbach (10 https://orcid.org/0000-0002-1980-5293

B. Giacomazzo (iD https://orcid.org/0000-0002-6947-4023

G. González (ib https://orcid.org/0000-0003-0199-3158

A. Grado (ib https://orcid.org/0000-0002-0501-8256

C.-J. Haster (1) https://orcid.org/0000-0001-8040-9807

I. S. Heng (iD https://orcid.org/0000-0002-1977-0019

D. E. Holz (i) https://orcid.org/0000-0002-0175-5064

V. Kalogera (ib https://orcid.org/0000-0001-9236-5469

D. Keitel (iD https://orcid.org/0000-0002-2824-626X

P. Koch (1) https://orcid.org/0000-0003-2777-5861

I. Kowalska (i) https://orcid.org/0000-0002-6569-3800

B. Krishnan (ib https://orcid.org/0000-0003-3015-234X

J. Lange (i) https://orcid.org/0000-0002-2450-1366

P. D. Lasky (1) https://orcid.org/0000-0003-3763-1386

K. Lee (i) https://orcid.org/0000-0003-3175-1336

C. O. Lousto (ib https://orcid.org/0000-0002-6400-9640

C. Messick (D) https://orcid.org/0000-0002-8230-3309

K. Mogushi (i) https://orcid.org/0000-0003-3746-2586

M. Obergaulinger (iD https://orcid.org/0000-0001-56641382

R. O’Shaughnessy (iD https://orcid.org/0000-0001-5832-

8517

B. J. Owen (10) https://orcid.org/0000-0003-3919-0780

C. Pankow (10) https://orcid.org/0000-0002-1128-3662

F. Pannarale (ib https://orcid.org/0000-0002-7537-3210

M. A. Papa (i) https://orcid.org/0000-0002-1007-5298

M. Pitkin (i) https://orcid.org/0000-0003-4548-526X

P. Raffai (iD https://orcid.org/0000-0001-7576-0141

Javed Rana (iD https://orcid.org/0000-0001-5605-1809

M. Razzano (ib) https://orcid.org/0000-0003-4825-1629

T. Regimbau (iD https://orcid.org/0000-0002-0631-1198

F. Ricci (1) https://orcid.org/0000-0001-5742-5980

P. M. Ricker (iD https://orcid.org/0000-0002-5294-0630

N. Sarin (i) https://orcid.org/0000-0003-2700-1030

C. Talbot (iD https://orcid.org/0000-0003-2053-5582

K. Ueno (1) https://orcid.org/0000-0003-0424-3045

G. Woan (iD https://orcid.org/0000-0003-0381-0394

Hang Yu (iD https://orcid.org/0000-0002-6011-6190

M. Zevin (ib https://orcid.org/0000-0002-0147-0835

\section{References}

Aasi, J., Abadie, J., Abbott, B. P., et al. 2014, CQGra, 31, 085014 Aasi, J., Abadie, J., Abbott, B. P., et al. 2015a, CQGra, 32, 074001 Aasi, J., Abadie, J., Abbott, B. P., et al. 2015b, ApJ, 813, 39

Abbott, B. P., Abbott, R., Abbott, T. D., et al. 2017a, PhRvD, 96, 062002 Abbott, B. P., Abbott, R., Abbott, T. D., et al. 2017b, CQGra, 34, 044001 Abbott, B. P., Abbott, R., Abbott, T. D., et al. 2017c, ApJL, 848, L13

Abbott, B. P., Abbott, R., Abbott, T. D., et al. 2017d, PhRvL, 119, 141101 Abbott, B. P., Abbott, R., Abbott, T. D., et al. 2017e, ApJL, 848, L12

Abbott, B. P., Abbott, R., Abbott, T. D., et al. 2017f, PhRvD, 95, 122003

Abbott, B. P., Abbott, R., Abbott, T. D., et al. 2017g, ApJL, 851, L16

Abbott, B. P., Abbott, R., Abbott, T. D., et al. 2018a, CQGra, 35, 065009

Abbott, B. P., Abbott, R., Abbott, T. D., et al. 2018b, PhRvD, 97, 102003

Abbott, B. P., Abbott, R., Abbott, T. D., et al. 2018c, PhRvL, 121, 161101

Abbott, B. P., Abbott, R., Abbott, T. D., et al. 2018d, LRR, 21, 3

Abbott, B. P., Abbott, R., Abbott, T. D., et al. 2019, PhRvX, 9, 011001

Acernese, F., Agathos, M., Agatsuma, K., et al. 2015, CQGra, 32, 024001

Ai, S., Gao, H., Dai, Z.-G., et al. 2018, ApJ, 860, 57

Alford, M. G., \& Schwenzer, K. 2014, ApJ, 781, 26

Alford, M. G., \& Schwenzer, K. 2015, MNRAS, 446, 3631

Antonucci, F., Astone, P., D'Antonio, S., Frasca, S., \& Palomba, C. 2008, CQGra, 25, 184015

Arras, P., Flanagan, E. E., Morsink, S. M., et al. 2003, ApJ, 591, 1129

Astone, P., Colla, A., D’Antonio, S., Frasca, S., \& Palomba, C. 2014, PhRvD, 90, 042002

Baiotti, L., \& Rezzolla, L. 2017, RPPh, 80, 096901

Barsotti, L., McCuller, L., Evans, M., \& Fritschel, P. 2018, The A+ Design Curve, Tech. Rep. LIGO-T1800042

Bauswein, A., Just, O., Janka, H.-T., \& Stergioulas, N. 2017, ApJL, 850, L34

Bondarescu, R., Teukolsky, S. A., \& Wasserman, I. 2009, PhRvD, 79, 104003

Cahillane, C., Betzwieser, J., Brown, D. A., et al. 2017, PhRvD, 96, 102001

Coulter, D. A., Foley, R. J., Kilpatrick, C. D., et al. 2017, Sci, 358, 1556

Covas, P. B., Effler, A., Goetz, E., et al. 2018, PhRvD, 97, 082002

Cutler, C. 2002, PhRvD, 66, 084025

Dall'Osso, S., Giacomazzo, B., Perna, R., \& Stella, L. 2015, ApJ, 798, 25

Dall'Osso, S., Shore, S. N., \& Stella, L. 2009, MNRAS, 398, 1869

Dall'Osso, S., Stella, L., \& Palomba, C. 2018, MNRAS, 480, 1353

Davis, D., Massinger, T. J., Lundgren, A. P., et al. 2019, CQGra, 36, 055011

Dooley, K. L., Leong, J. R., Adams, T., et al. 2016, CQGra, 33, 075009

Driggers, J. C., Vitale, S., Lundgren, A. P., et al. 2019, PhRvD, 99, 042001

Freedman, W. L., Madore, B. F., Gibson, B. K., et al. 2001, ApJ, 553, 47

Geng, J.-J., Dai, Z.-G., Huang, Y.-F., et al. 2018, ApJL, 856, L33

Granot, J., Gill, R., Guetta, D., \& De Colle, F. 2018, MNRAS, 481, 1597

Granot, J., Guetta, D., \& Gill, R. 2017, ApJL, 850, L24

Hild, S., Abernathy, M., Acernese, F., et al. 2011, CQGra, 28, 094013

Hjorth, J., Levan, A. J., Tanvir, N. R., et al. 2017, ApJL, 848, L31

Jaranowski, P., Królak, A., \& Schutz, B. F. 1998, PhRvD, 58, 063001

Johnson-McDaniel, N. K., \& Owen, B. J. 2013, PhRvD, 88, 044004

Kasen, D., Metzger, B., Barnes, J., Quataert, E., \& Ramirez-Ruiz, E. 2017, Natur, 551, 80

Keitel, D. 2016, PhRvD, 93, 084024

Krishnan, B., Sintes, A. M., Papa, M. A., et al. 2004, PhRvD, 70, 082001

Lasky, P. D., Sarin, N., \& Sammut, L. 2017, Long-duration Waveform Models for Millisecond Magnetars Born in Binary Neutron Star Mergers, Tech. Rep. LIGO-T1700408

Lee, M. G., Kang, J., \& Im, M. 2018, ApJL, 859, L6

Li, S.-Z., Liu, L.-D., Yu, Y.-W., \& Zhang, B. 2018, ApJL, 861, L12

Margalit, B., \& Metzger, B. D. 2017, ApJL, 850, L19

Matsumoto, T., Ioka, K., Kisaka, S., \& Nakar, E. 2018, ApJ, 861, 55

Messenger, C., Bulten, H. J., Crowder, S. G., et al. 2015, PhRvD, 92, 023006

Miller, A., Astone, P., D’Antonio, S., et al. 2018, PhRvD, 98, 102004

Oliver, M., Keitel, D., \& Sintes, A. M. 2019, arXiv:1901.01820

Owen, B. J., Lindblom, L., Cutler, C., et al. 1998, PhRvD, 58, 084020

Palomba, C. 2001, A\&A, 367, 525

Palomba, C., Astone, P., \& Frasca, S. 2005, CQGra, 22, S1255

Piro, A. L., Giacomazzo, B., \& Perna, R. 2017, ApJL, 844, L19

Pooley, D., Kumar, P., Wheeler, J. C., \& Grossan, B. 2018, ApJL, 859, L23

Prix, R. 2009, in Neutron Stars and Pulsars, ed. W. Becker (Berlin: Springer), 651 
Prix, R., Giampanis, S., \& Messenger, C. 2011, PhRvD, 84, 023007

Radice, D., Perego, A., Zappa, F., \& Bernuzzi, S. 2018, ApJL, 852, L29

Ravi, V., \& Lasky, P. D. 2014, MNRAS, 441, 2433

Reisenegger, A. 2009, A\&A, 499, 557

Rezzolla, L., Most, E. R., \& Weih, L. R. 2018, ApJL, 852, L25

Riles, K. 2017, MPLA, 32, 1730035

Sakai, S., Mould, J. R., Hughes, S. M. G, et al. 2000, ApJ, 529, 698

Sarin, N., Lasky, P. D., Sammut, L., \& Ashton, G. 2018, PhRvD, 98, 043011

Sathyaprakash, B., Abernathy, M., Acernese, F., et al. 2012, CQGra, 29, 124013

Shapiro, S. L., \& Teukolsky, S. A. 1983, Black Holes, White Dwarfs, and Neutron Stars: The Physics of Compact Objects (New York: Wiley)

Sintes, A. M., \& Krishnan, B. 2007, Hough Search with Improved Sensitivity, Tech. Rep. LIGO-T070124
Sun, L., \& Melatos, A. 2018, arXiv:1810.03577

Sun, L., Melatos, A., Lasky, P., Chung, C., \& Darman, N. 2016, PhRvD, 94, 082004

Sun, L., Melatos, A., Suvorova, S., Moran, W., \& Evans, R. 2018, PhRvD, 97, 043013

Suvorova, S., Sun, L., Melatos, A., Moran, W., \& Evans, R. J. 2016, PhRvD, 93, 123009

Thrane, E., \& Coughlin, M. 2013, PhRvD, 88, 083010

Thrane, E., Kandhasamy, S., Ott, C. D., et al. 2011, PhRvD, 83, 083004

Thrane, E., Mandic, V., \& Christensen, N. 2015, PhRvD, 91, 104021

van Putten, M. H. P. M., \& Della Valle, M. 2018, MNRAS, 482, L46

Viterbi, A. 1967, ITIT, 13, 260

Walsh, S., Pitkin, M., Oliver, M., et al. 2016, PhRvD, 94, 124010

Yu, Y.-W., Liu, L.-D., \& Dai, Z.-G. 2018, ApJ, 861, 114

Zhu, S. J., Papa, M. A., Eggenstein, H.-B., et al. 2016, PhRvD, 94, 082008 